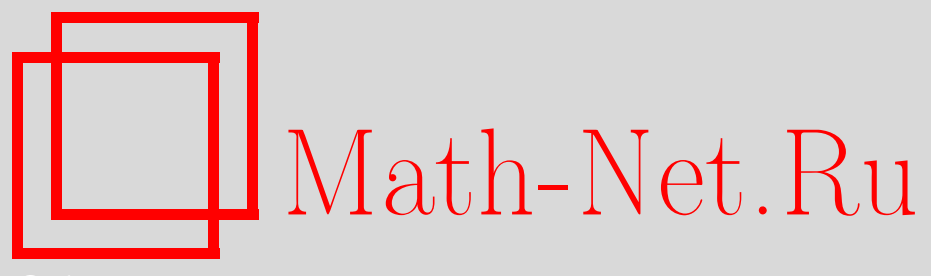

Г. Е. Иванов, Точные оценки модулей непрерывности метрической проекции на слабо выпуклые множества, Изв. РАН. Сер. матем., 2015, том 79, выпуск 4, 27-56

DOI: https://doi.org/10.4213/im8246

Использование Общероссийского математического портала Math-Net.Ru подразумевает, что вы прочитали и согласны с пользовательским соглашением http://www . mathnet.ru/rus/agreement

Параметры загрузки:

IP: 54.89 .56 .158

26 апреля 2023 г., 16:01:26

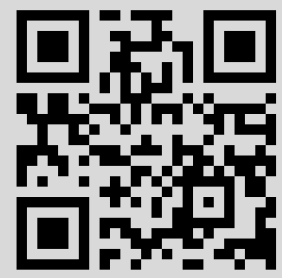




\title{
Г. Е. Иванов \\ Точные оценки модулей непрерывности метрической проекции на слабо выпуклые множества
}

\begin{abstract}
Рассматривается зависимость метрической проекции от следующих трех параметров: проектируемой точки, множества, на которое происходит проектирование, и нормы (вообще говоря, несимметричной), определяющей метрику. Получены точные оценки модулей непрерывности метрической проекции на выпуклые и слабо выпуклые множества в банаховом пространстве. Также даны оценки этих модулей через модули выпуклости и гладкости пространства (или квазишара).

Библиография: 27 наименований.
\end{abstract}

Ключевые слова: метрическая проекция, слабо выпуклые множества.

DOI: $10.4213 / \operatorname{im} 8246$

\section{$\S 1$. Введение}

Метрической проекиией заданной точки на заданное множество называется множество точек, наилучшим образом приближающих заданную точку элементом заданного множества в смысле рассматриваемой метрики. Оператор метрической проекции широко используется в функциональном анализе, методах аппроксимации и оптимизации. Модуль непрерывности этого оператора показывает, насколько метрическая проекция чувствительна к малым варьированиям параметров. Эта важная характеристика входит в оценки скоростей сходимости некоторых численных методов, например метода проекции градиента [1].

Как известно, метрическая проекция на выпуклые замкнутые множества в гильбертовом пространстве однозначна и удовлетворяет условию Липшица с константой 1 относительно проектируемой точки. Оценками модулей непрерывности метрической проекции на выпуклые замкнутые множества в банаховых пространствах занимались Б. Бьёрнстол [2], [3], В. И. Бердышев [4]-[6], Я. И. Альбер и А. И. Нотик [7], [8], Ж. Пено [9] и другие авторы.

Метрическая проекция на слабо выпуклое замкнутое множество однозначна и непрерывна на $r$-окрестности этого множества, причем величина $\frac{1}{r}$ характеризует степень невыпуклости множества. Класс слабо выпуклых множеств чрезвычайно широк и включает в себя все гладкие (в определенном смысле) и все выпуклые множества. Множества этого класса изучались многими авторами, которые называли их по-разному. В конечномерном евклидовом пространстве Г. Федерер [10] называл такие множества множествами положительной достижимости, а ЖК.-Ф. Виаль [11] называл их слабо выпуклыми множествами.

Работа выполнена при финансовой поддержке РФФИ (грант № 13-01-00295). 
В гильбертовом пространстве Ф. Кларк, Р. Штерн и П. Воленски [12] изучали эти множества под именем проксимально гладких множеств. Ф. Бернард, Л. Тибо и Н. Златева [13], [14] рассматривали свойства таких множеств в банаховых пространствах, называя эти множества равномерно прокс-регулярными. М. В. Балашов и Г. Е. Иванов [15] исследовали эти множества, называя их множествами, удовлетворяющими опорному условию слабой выпуклости.

Следующим важным шагом на пути обобщения этих определений является рассмотрение соответствующего класса множеств в пространствах с несимметричной нормой. В. Гончаров и Ф. Перейра [16], [17], а также Ж. Коломбо, В. Гончаров и Б. Мордухович [18], рассматривая задачу о наилучшем приближении относительно несимметричной нормы, продемонстрировали применения этой задачи в теории оптимального управления. В работах [19], [20] показано, что изучение класса слабо выпуклых множеств относительно несимметричной полунормы (или, что то же самое, относительно несимметричного квазишара) позволяет получить новые результаты о корректности задач оптимизации.

В работе [21] получены некоторые локальные оценки модуля непрерывности метрической проекции на аппроксимативно компактное множество с использованием локального радиуса кривизны этого множества. Указанный радиус кривизны по смыслу близок к параметру $r$ в определении $r$-прокс-регулярных множеств, рассмотренном в работах [13], [14]. В работе [14], в частности, показано, что если модуль выпуклости пространства имеет степенной порядок со степенью $q$, а модуль гладкости пространства - со степенью $s>1$, то метрическая проекция на $r$-прокс-регулярное множество $A$ как функция проектируемой точки является локально гёльдеровой с показателем $\frac{1}{q}$ в $\frac{r}{2}$-окрестности множества $A$. Из оценок, полученных в настоящей работе, следует, что при тех же условиях показатель в условии Гёльдера может быть увеличен до $\frac{s}{q}$. Отметим, что для метрической проекции на подпространство показатель Гёльдера $\frac{s}{q}$ (в виде оценки через суперпозицию функции, обратной к модулю выпуклости и модулю гладкости пространства) был получен в [2], [3].

В настоящей работе также показано, что метрическая проекция на слабо выпуклое множество как функция этого множества и нормы пространства удовлетворяет условию Гёльдера с показателем $\frac{1}{q}$. Подобные результаты для выпуклых множеств были получены в [4]-[6].

Далее в $\S 2$ даны определения для стандартной метрики, а в $\S 3,4$ - соответствующие результаты. В $\S 5$ приведены определения, а в $\S 6$ - формулировки результатов, относящихся к метрике, определяемой несимметричной нормой или квазишаром. В $\S 7$ помещены вспомогательные результаты. В $\S 8$ доказаны результаты из $\S 6$ и, как следствие, получены результаты из $\S 3,4$.

\section{§ 2. Обозначения и определения}

Пусть $E$ - вещественное линейное нормированное пространство. Через int $A$ и $\partial A$ будем обозначать соответственно внутренность и границу множества $A \subset E$, значение функционала $f \in E^{*}$ на векторе $x \in E$ - через $\langle f, x\rangle$, замкнутый шар в $E$ с радиусом $R>0$ и центром $c \in E-$ через $\mathfrak{B}_{R}(c)$. Положим $\mathfrak{B}_{R}:=\mathfrak{B}_{R}(0)$.

Диаметром множества $A$ называется

$$
\operatorname{diam} A=\sup _{x, y \in A}\|x-y\|
$$


Расстояние от точки $x \in E$ до множества $A \subset E$ определяется равенством

$$
d(x, A)=\inf _{a \in A}\|x-a\| .
$$

При $r>0$ определим $r$-окрестность множества $A \subset E$ формулой

$$
O(A, r)=\{x \in E \mid d(x, A)<r\} .
$$

Множеством наилучших приближений или метрической проекцией точки $x \in E$ на множество $A \subset E$ называется множество

$$
P(x, A)=\{a \in A \mid\|x-a\|=d(x, A)\} .
$$

Конусом проксимальных нормалей множества $A \subset E$ в точке $a \in A$ называется

$$
N(a, A)=\{z \in E \mid \exists t>0: a \in P(a+t z, A)\} .
$$

Пусть $r>0$. Множество $A \subset E$ называется равномерно $r$-прокс-регулярным [13], если

$$
P(a+r z, A)=\{a\} \quad \forall a \in A, \quad \forall z \in N(a, A):\|z\|<1 .
$$

Через Conv обозначим класс выпуклых подмножеств пространства $E$, а через $\mathcal{P} \mathcal{R}(r)$ - класс равномерно $r$-прокс-регулярных его подмножеств. Легко видеть, что если пространство $E$ строго выпукло, то Conv $\subset \mathcal{P} \mathcal{R}(r)$ для любого $r>0$.

Пусть заданы неотрицательные числа $t, d$ и класс $\mathfrak{F}$ подмножеств $A$ пространства $E$ таких, что $P(x, A) \neq \varnothing$ для любой точки $x \in E$, удовлетворяющей равенству $d(x, A)=d$.

Модулем непрерывности метрической проекиии (относительно варъирования проектируемой точки) называется

$$
\omega(t, d, \mathfrak{F})=\sup _{A \in \mathfrak{F}} \sup _{\substack{x_{1}, x_{2} \in E:\left\|x_{1}-x_{2}\right\| \leqslant t \\ d\left(x_{1}, A\right)=d \\ P\left(x_{2}, A\right) \neq \varnothing}} \sup _{\substack{a_{1} \in P\left(x_{1}, A\right) \\ a_{2} \in P\left(x_{2}, A\right)}}\left\|a_{1}-a_{2}\right\| .
$$

Расстоянием Хаусдорфа между множествами $A \subset E$ и $B \subset E$ называется

$$
h(A, B)=\max \left\{\sup _{x \in A} d(x, B), \sup _{x \in B} d(x, A)\right\} .
$$

Модулем непрерывности метрической проекции относителъно варъирования множества называется

$$
\theta(t, d, \mathfrak{F})=\sup _{\substack{A_{1} \in \mathcal{F}, A_{2} \subset E: \\ h\left(A_{1}, A_{2}\right) \leqslant t}} \sup _{\substack{x \in E: d\left(x, A_{1}\right)=d \\ P\left(x, A_{2}\right) \neq \varnothing}} \sup _{\substack{a_{1} \in P\left(x, A_{1}\right) \\ a_{2} \in P\left(x, A_{2}\right)}}\left\|a_{1}-a_{2}\right\| .
$$

Определим модуль непрерывности метрической проекции относительно варьирования нормы. Пусть $n: E \rightarrow[0,+\infty)$ - некоторая норма пространства $E$, эквивалентная исходной. Расстоянием от нормы $n(\cdot)$ до исходной нормы $\|\cdot\|$ будем называть

$$
D(n(\cdot),\|\cdot\|)=\sup \{|1-n(x)| \mid x \in E:\|x\|=1\} .
$$


Расстояние от точки $x \in E$ до множества $A \subset E$ и метрическая проекция точки $x$ на множество $A$ относительно нормы $n(\cdot)$ определяются соответственно равенствами

$$
\begin{gathered}
d^{n(\cdot)}(x, A)=\inf _{a \in A} n(x-a), \\
P^{n(\cdot)}(x, A)=\left\{a \in A \mid n(x-a)=d^{n(\cdot)}(x, A)\right\} .
\end{gathered}
$$

Модулем непрерывности метрической проекции относительно варьирования нормы называется

$$
\nu(t, d, \mathfrak{F})=\sup _{n(\cdot): D(n(\cdot),\|\cdot\|) \leqslant t} \sup _{\begin{array}{c}
A \in \mathfrak{F}, x \in E: \\
d(x, A)=d \\
P^{n(\cdot)}(x, A) \neq \varnothing
\end{array}} \sup _{\substack{a \in P(x, A) \\
a^{\prime} \in P^{n(\cdot)}(x, A)}}\left\|a-a^{\prime}\right\|,
$$

где $n(\cdot)$ - произвольная норма пространства $E$, удовлетворяющая неравенству $D(n(\cdot),\|\cdot\|) \leqslant t$

Модуль выпуклости пространства $E$ определяется равенством

$$
\delta(t)=\inf \left\{1-\frac{\|x+y\|}{2} \mid x, y \in \mathfrak{B}_{1},\|x-y\| \geqslant t\right\}, \quad t \in[0,2] .
$$

Множество $M \subset E$ называется строго выпуклым, если для любых двух различных точек $x, y \in M$ справедливо включение $\frac{x+y}{2} \in \operatorname{int} M$. Пространство $E$ называется строго выпуклым, если единичный шар $\mathfrak{B}_{1}$ является строго выпуклым множеством. Пространство $E$ называется равномерно выпуклым, если $\delta(t)>0$ при всех $t \in(0,2]$. Легко видеть, что каждое равномерно выпуклое пространство строго выпукло. Известно, что модуль выпуклости любого равномерно выпуклого пространства является непрерывной строго возрастающей функцией. Обратную функцию к модулю выпуклости будем обозначать через $\delta^{-1}(\cdot)$. Будем говорить, что модуль выпуклости пространства $E$ nоряд$\kappa a q$, если для некоторого $C_{1}>0$ при всех $t \in(0,2]$ справедливо неравенство $\delta(t) \geqslant C_{1} t^{q}$. Очевидно, если модуль выпуклости пространства $E$ порядка $q$, то пространство $E$ равномерно выпукло.

Модулем гладкости пространства $E$ называется

$$
\varrho(t)=\frac{1}{2} \sup \{\|x+y\|+\|x-y\|-2 \mid x, y \in E:\|x\|=1,\|y\|=t\}, \quad t \geqslant 0 .
$$

Пространство $E$ называется равномерно гладким, если $\lim _{t \rightarrow+0} \frac{\varrho(t)}{t}=0$. Будем говорить, что модуль гладкости пространства $E$ порядка $s$, если для некоторого $C_{2}>0$ при всех $t>0$ справедливо неравенство $\varrho(t) \leqslant C_{2} t^{s}$.

Из равенства (2.9) следует, что для любого пространства $E$ при всех $t \geqslant 0$ справедливо неравенство $\varrho(t) \leqslant t$. Поэтому модуль гладкости любого (не только равномерно выпуклого) пространства $E$ по меньшей мере порядка $s=1$. Если модуль гладкости пространства $E$ порядка $s>1$, то пространство $E$ равномерно гладкое. 
По теореме Дея-Нордлендера [22] модуль выпуклости $\delta^{E}(\cdot)$ и модуль гладкости $\varrho^{E}(\cdot)$ любого нормированного пространства $E$ связаны с модулем выпуклости $\delta^{H}(\cdot)$ и модулем гладкости $\varrho^{H}(\cdot)$ гильбертова пространства $H$ следующими соотношениями:

$$
\begin{gathered}
\delta^{E}(t) \leqslant \delta^{H}(t)=1-\sqrt{1-\frac{t^{2}}{4}} \quad \forall t \in[0,2], \\
\varrho^{E}(t) \geqslant \varrho^{H}(t)=\sqrt{1+t^{2}}-1 \quad \forall t \geqslant 0 .
\end{gathered}
$$

Поэтому, если модуль выпуклости нормированного пространства $E$ порядка $q$, а его модуль гладкости - порядка $s \geqslant 1$, то

$$
1 \leqslant s \leqslant 2 \leqslant q
$$

Через $\operatorname{Lin}_{2}$ обозначим класс двумерных линейных подпространств пространства $E$. Шириной множества $A \subset L$ относительно подпространства $L \in \operatorname{Lin}_{2}$ называется величина

$$
w_{L}(A)=\inf _{f \in L^{*}:\|f\|=1} \sup _{a_{1}, a_{2} \in A}\left\langle f, a_{1}-a_{2}\right\rangle,
$$

где $L^{*}$ - пространство, сопряженное к пространству $L$, норма которого индуцирована нормой пространства $E ;\|f\|$ - норма функционала в $L^{*}$.

В. И. Бердышев [4], [6] получил точные оценки модуля $\omega(t, d$, Conv) (cм. (2.4)) через введенную им следующую характеристику пространства $E$ :

$$
\Omega(t)=\sup _{a \in \mathfrak{B}_{1}} \sup _{\substack{L \in \operatorname{Lin}_{2}: \\ w_{L}\left(L \cap \mathfrak{B}_{1}(-a)\right) \leqslant t}} \operatorname{diam}\left(L \cap \mathfrak{B}_{1}(-a)\right), \quad t \geqslant 0 .
$$

Для получения точной оценки модуля $\omega(t, d, \mathcal{P} \mathcal{R}(r))$ удобно использовать следующий модуль пространства $E$ :

$$
\begin{aligned}
\Phi(t)=\sup \{\|x\| \mid x, y, z \in E:\|z+x\|=\|z-x\|=1 \\
\|z+y\| \geqslant 1,\|z-y\| \geqslant 1,\|y\| \leqslant t\}, \quad t \geqslant 0 .
\end{aligned}
$$

Далее будет показано, что функции $\Omega(t)$ и $\Phi(t)$ одного порядка при $t \rightarrow+0$.

Пусть на интервале $\left(0, t_{0}\right)$ (где $t_{0}>0$ ) заданы возрастающие функции $\varphi:\left(0, t_{0}\right) \rightarrow(0,+\infty)$ и $\psi:\left(0, t_{0}\right) \rightarrow(0,+\infty)$. Будем говорить, что функции $\varphi(t)$ и $\psi(t)$ одного порядка, и записывать $\varphi(t) \asymp \psi(t)$ при $t \rightarrow+0$, если существуют числа $C \geqslant 1$ и $t_{1} \in\left(0, \frac{t_{0}}{C}\right)$ такие, что

$$
\varphi(t) \leqslant C \psi(C t), \quad \psi(t) \leqslant C \varphi(C t) \quad \forall t \in\left(0, t_{1}\right) .
$$

\section{§ 3. Оценки модулей непрерывности метрической проекции относительно варьирования проектируемой точки}

В.И. Бердышев [4], [6] получил следующие оценки модуля непрерывности метрической проекции (2.4) на выпуклые множества через функцию $\Omega(\cdot)$ (см. (2.14)). 
ПреДЛОЖЕНИЕ 3.1 [6, теорема 2.1]. Для любого строго выпуклого пространства Е справедливы следующие оченки:

$$
\frac{d}{2} \Omega\left(\frac{t}{3 d}\right)-\frac{3}{2} t \leqslant \omega(t, d, \text { Conv }) \leqslant d \Omega\left(\frac{2 t}{d}\right)+2 t \quad \forall d>0, \quad \forall t \in(0, d) .
$$

Следующая теорема дает оценки модуля непрерывности метрической проекции на прокс-регулярные множества.

Tеорема 3.1. Пусть пространство Е строго выпукло. Тогда для любых $r>0, d \in(0, r), t \in(0, d)$ справедливы оценки

$$
d \cdot \Phi\left(\frac{t}{8 d}\right)-\frac{9}{8} t \leqslant \omega(t, d, \mathrm{Conv}) \leqslant \omega(t, d, \mathcal{P} \mathcal{R}(r)) \leqslant 2 d \cdot \Phi\left(\frac{t}{\min \{d, r-d\}}\right)+2 t .
$$

СЛЕДСТвИЕ 3.1. Для любого строго выпуклого пространства Е и для любых фиксированных чисел $r>0, d \in(0, r)$ имеют место соотношения

$$
\omega(t, d, \mathcal{P} \mathcal{R}(r)) \asymp \omega(t, d, \mathrm{Conv}) \asymp \Phi(t), \quad t \rightarrow+0 .
$$

Теорема 3.1 позволяет свести оценку модуля непрерывности метрической проекции к оценке модуля $\Phi$. Следующие три леммы дают различные оценки модуля $\Phi$.

Лемма 3.1. Для любого строго выпуклого пространства Е справедливъ оценки

$$
\frac{1}{2} \Omega(t) \leqslant \Phi(t) \leqslant \Omega(16 t)+9 t \quad \forall t \in\left(0, \frac{1}{8}\right) .
$$

Лемма 3.2. Для любого равномерно выпуклого пространства Е справедлива следующая оченка модуля Ф через модули выпуклости и гладкости пространства E:

$$
2 \Phi(t) \leqslant \delta^{-1}(\varrho(2 t)) \quad \forall t \in\left(0, \frac{1}{2}\right] .
$$

В общем случае оценка (3.2) не является точной, однако для лебеговых пространств она точна по порядку, что вытекает из следующей леммы.

Лемма 3.3. Пусть $p \in(1,+\infty), E=L_{p}, \operatorname{dim} E \geqslant 3$. Тогда

$$
\Phi(t) \asymp \delta^{-1}(\varrho(t)) \asymp t^{\alpha}, \quad t \rightarrow+0,
$$

где $\alpha=\min \left\{\frac{p}{2}, \frac{2}{p}\right\}$.

В работе [13, теорема 6.2] получена следующая характеризация класса равномерно $r$-прокс-регулярных множеств.

ПРЕДЛОЖЕНИЕ 3.2. Пусть модули выпуклости и гладкости пространства $E$ порядков q us $>1$ соответственно. Пусть множество $A \subset E$ замкнуто u $r>0$. Тогда следующие условия эквивалентны:

(i) $A \in \mathcal{P} \mathcal{R}(r)$;

(ii) оператор метрической проекиии $x \mapsto P(x, A)$ однозначен и непрерывен на множестве $O(A, r)$. 
Следующее предложение дает оценку порядка модуля непрерывности оператора метрической проекции на равномерно $r$-прокс-регулярное множество.

ПредлОЖеНИЕ 3.3 [14, теорема 9.1]. Пусть модули выпуклости и гладкости пространства Е порядков $q u s>1$ соответственно. Пусть числа $r, r^{\prime}$ удовлетворяют неравенствам $0<r^{\prime}<\frac{r}{2} u A \in \mathcal{P} \mathcal{R}(r)$. Тогда для любого числа $R>0$ оператор метрической проекиии $x \mapsto P(x, A)$ удовлетворяет условию Гёльдера на множестве $O\left(A, r^{\prime}\right) \cap \mathfrak{B}_{R}$ с показателем $\frac{1}{q}$.

Показатель Гёльдера в предложении 3.3 может быть улучшен, что показывает следующая теорема.

Теорема 3.2. Пусть модуль выпуклости пространства Е порядка q, а модуль гладкости $E$ порядка $s \geqslant 1$. Пусть $A \in \mathcal{P} \mathcal{R}(r)$ при некотором $r>0$. Тогда для любого $r^{\prime} \in(0, r)$ оператор метрической проекиии $x \mapsto P(x, A)$ удовлетворяет условию Гёльдера на множестве $O\left(A, r^{\prime}\right)$ с показателем $\frac{s}{q}$.

\section{§4. Оценки модулей непрерывности метрической проекции относительно варьирования множества и нормы}

Рассмотрим зависимость метрической проекции от множества, на которое происходит проектирование. Соответствующий модуль непрерывности определяется формулой (2.5).

ПРедлОЖениЕ 4.1 [6, предложение 2.1]. Пусть пространство Е равномерно выпукло. Тогда

$$
\frac{d}{2} \delta^{-1}\left(\frac{t}{8 d}\right)-\frac{t}{2} \leqslant \theta(t, d, \text { Conv }) \leqslant d \delta^{-1}\left(\frac{t}{2 d}\right)+t \quad \forall d>0, \quad \forall t \in(0, d) .
$$

Теорема 4.1. Пусть пространство Е равномерно выпукло. Тогда

$$
\theta(t, d, \mathcal{P} \mathcal{R}(r)) \leqslant d \delta^{-1}\left(\frac{t}{\min \{d, r-d\}}\right)+t \quad \forall r>0, \quad \forall d \in(0, r), \quad \forall t \in(0, d) .
$$

СлЕДСТвИЕ 4.1. Если пространство Е равномерно выпукло, то для любъх фиксированных чисел $r>0 u d \in(0, r)$ справедливы соотношения

$$
\theta(t, d, \mathcal{P} \mathcal{R}(r)) \asymp \theta(t, d, \mathrm{Conv}) \asymp \delta^{-1}(t), \quad t \rightarrow+0 .
$$

СлеДСТВИЕ 4.2. Пусть модулъ выпуклости пространства Е порядка $q, u$ пусть $x \in E, r>0$. Тогда для любого $r^{\prime} \in(0, r)$ оператор метрической проекиии $A \mapsto P(x, A)$ как функиия множества А удовлетворяет условию Гёльдера на множестве $\left\{A \in \mathcal{P} \mathcal{R}(r) \mid d(x, A) \leqslant r^{\prime}\right\}$ с показателем $\frac{1}{q}$.

Теперь рассмотрим зависимость метрической проекции от нормы, определяющей метрику. Соответствующий модуль непрерывности определяется формулой (2.7).

ПредЛОЖЕНИЕ 4.2 [6, П. 3.3, теорема 2.1]. Пусть пространство Е равномерно выпукло. Тогда

$$
\frac{d}{2} \delta^{-1}\left(\frac{t}{2 d}\right) \leqslant \nu(t, d, \text { Conv }) \leqslant d \delta^{-1}\left(\frac{3 t}{2 d}\right)+3 t \quad \forall d>0, \quad \forall t \in(0, d) .
$$


Теорема 4.2. Для любого равномерно выпуклого пространства Е и для любых чисел $r>0, d \in(0, r)$ u $t \in\left(0, \frac{1}{2}\right)$ справедлива оченка

$$
\nu(t, d, \mathcal{P} \mathcal{R}(r)) \leqslant d \delta^{-1}\left(\frac{d t}{\min \{(1-t) d,(1-2 t)(r-d)\}}\right)+\frac{2 d t}{1-2 t} .
$$

СлЕДСТвИЕ 4.3. Если пространство Е равномерно выпукло, то для любых фиксированных чисел $r>0 u d \in(0, r)$ справедливы соотношения

$$
\begin{gathered}
\nu(t, d, \mathcal{P} \mathcal{R}(r)) \asymp \nu(t, d, \mathrm{Conv}) \asymp \delta^{-1}(t), \quad t \rightarrow+0 . \\
\S 5 . \text { Метрическая проекция } \\
\text { и слабо выпуклые множества относительно квазишара }
\end{gathered}
$$

Квазишаром называется выпуклое замкнутое множество $M \subset E$ такое, что $0 \in \operatorname{int} M$.

ЗАмечАнИЕ 5.1. Квазишар $M$ является единичным шаром относительно некоторой нормы, эквивалентной исходной, тогда и только тогда, когда он ограничен относительно исходной нормы и симметричен (т. е. $-M=M)$.

Функиионал Минковского квазишара $M \subset E$ определяется формулой

$$
\mu_{M}(x)=\inf \left\{\begin{array}{l|l}
t>0 & \frac{x}{t} \in M
\end{array}\right\}, \quad x \in E .
$$

Через $d_{M}(x, A)$ обозначим $M$-расстояние от точки $x \in E$ до множества $A \subset E$ :

$$
d_{M}(x, A)=\inf _{a \in A} \mu_{M}(x-a) .
$$

ЗАмечАниЕ 5.2. Если в качестве квазишара $M$ взять единичный шар $\mathfrak{B}_{1}$, то функционал Минковского квазишара $M$ будет совпадать с нормой, а $M$-расстояние - с обычным расстоянием:

$$
\mu_{\mathfrak{B}_{1}}(x)=\|x\|, \quad d_{\mathfrak{B}_{1}}(x, A)=d(x, A) \quad \forall x \in E, \quad \forall A \subset E .
$$

ЗАмЕчАниЕ 5.3. По определению для любого квазишара $M$ найдется число $\sigma>0$ такое, что $\mathfrak{B}_{\sigma} \subset M$. При этом функционал Минковского $\mu_{M}(\cdot)$ удовлетворяет условию Липшица на $E$ с константой $\frac{1}{\sigma}$. Поэтому для любого множества $A \subset E$ функция $d_{M}(\cdot, A)$ удовлетворяет условию Липшица на $E$ с той же константой.

Функционал $\mu: E \rightarrow[0,+\infty)$ называется несимметричной нормой, если он положительно однороден:

$$
\mu(t x)=t \mu(x) \quad \forall t \geqslant 0, \quad \forall x \in E,
$$

субаддитивен:

$$
\mu(x+y) \leqslant \mu(x)+\mu(y) \quad \forall x, y \in E,
$$

и $\mu(x)>0$ для любого $x \in E \backslash\{0\}$. 
ЗАмечАнИЕ 5.4. Функция $\mu: E \rightarrow[0,+\infty)$, удовлетворяющая условию Липшица на $E$, является несимметричной нормой, эквивалентной исходной норме пространства $E$, тогда и только тогда, когда $\mu(\cdot)$ является функционалом Минковского некоторого ограниченного квазишара.

Задача нахождения элемента наилучшего приближения относительно несимметричной нормы (или, что то же самое, - относительно ограниченного квазишара $M)$ рассматривалась в работах [16]-[18], [20], [23]. Эта задача эквивалентна задаче быстродействия для динамической системы с постоянной динамикой.

Для заданных квазишара $M \subset E$, множества $A \subset E$ и точки $x \in E$ множеством элементов наилучшего приближения, или, что то же самое, $M$-проекиией точки $x$ на множество $A$, называется

$$
P_{M}(x, A)=\left\{a \in A \mid \mu_{M}(x-a)=d_{M}(x, A)\right\} .
$$

Конусом $M$-нормалей множества $A \subset E$ в точке $a \in A$ называется

$$
N_{M}(a, A)=\left\{z \in E \mid \exists t>0: a \in P_{M}(a+t z, A)\right\} .
$$

ЗАмЕчАниЕ 5.5. В силу замечания 5.2 справедливы следующие равенства:

$$
P_{\mathfrak{B}_{1}}(x, A)=P(x, A), \quad N_{\mathfrak{B}_{1}}(a, A)=N(a, A) .
$$

Множество $A \subset E$ называется слабо выпуклым относительно квазишара $M \subset E$, если

$$
a \in P_{M}(a+z, A) \quad \forall a \in A, \quad \forall z \in N_{M}(a, A): \mu_{M}(z) \leqslant 1 .
$$

Через $\mathcal{W C}(M)$ обозначим класс множеств $A \subset E$, слабо выпуклых относительно квазишара $M$.

ЗАмечАниЕ 5.6. Для любого квазишара $M$ справедливо включение Conv $\subset$ $\mathcal{W C}(M)$.

ЗАмечАниЕ 5.7. Если пространство $Е$ строго выпукло, то

$$
\mathcal{P} \mathcal{R}(r)=\mathcal{W C}\left(\mathfrak{B}_{r}\right) \quad \forall r>0 .
$$

Приведем определения модулей непрерывности $M$-проекции.

Пусть заданы неотрицательные числа $t, d$, квазишар $M$ и некоторый класс $\mathfrak{F}$ подмножеств $A$ пространства $E$ таких, что $P_{M}(x, A) \neq \varnothing$ для любой точки $x \in E$, удовлетворяющей равенству $d_{M}(x, A)=d$.

Модулем непрерьвности $M$-проекиии (относительно варьирования точки) называется

$$
\omega_{M}(t, d, \mathfrak{F})=\sup _{A \in \mathfrak{F}} \sup _{\begin{array}{c}
x_{1}, x_{2} \in E: \\
\left\|x_{1}-x_{2}\right\| \leqslant t \\
d_{M}\left(x_{1}, A\right)=d \\
P_{M}\left(x_{2}, A\right) \neq \varnothing
\end{array}} \sup _{\substack{a_{1} \in P_{M}\left(x_{1}, A\right) \\
a_{2} \in P_{M}\left(x_{2}, A\right)}}\left\|a_{1}-a_{2}\right\| .
$$

Расстоянием Хаусдорфа между множествами $A \subset E$ и $B \subset E$ относителъно квазишара $M$ называется

$$
h_{M}(A, B)=\max \left\{\sup _{x \in A} d_{M}(x, B), \sup _{x \in B} d_{M}(x, A)\right\} .
$$


Модулем непрерывности М-проекиии относительно варъирования множества называется

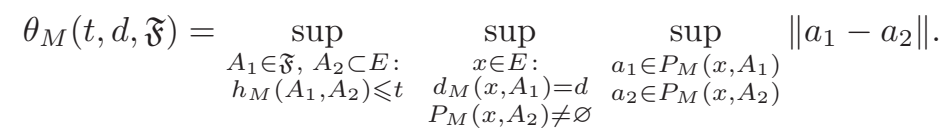

Модулем непрерывности $M$-проекиии относительно варъирования квазишара называется

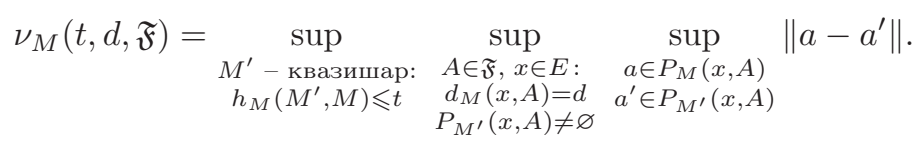

Модулем выпуклости квазишара $M$ называется

$$
\delta_{M}(t)=\inf \left\{1-\frac{\mu_{M}(x+y)}{2} \mid x, y \in M,\|x-y\| \geqslant t\right\}, \quad t \in(0, \operatorname{diam} M) .
$$

Квазишар $M$ называется равномерно выпуклым, если $\delta_{M}(t)>0$ при всех $t>0$. Известно [24], [25], что любой равномерно выпуклый квазишар ограничен и модуль выпуклости такого квазишара является непрерывной строго возрастающей функцией, поэтому в этом случае существует обратная функция $\delta_{M}^{-1}$.

ЗАмЕчАниЕ 5.8. Если пространство $E$ содержит равномерно выпуклый квазишар $M$, то множество $B=M \cap(-M)$ будет равномерно выпуклым. При этом $B$ - единичный шар относительно некоторой нормы, эквивалентной исходной норме $E$. Поэтому любое нормированное пространство $E$, содержащее равномерно выпуклый квазишар, допускает эквивалентную равномерно выпуклую норму, т. е. является суперрефлексивным.

Модуль гладкости квазишара $M$ определим формулой

$$
\varrho_{M}(t)=\sup \left\{\frac{\mu_{M}(x+y)+\mu_{M}(x-y)}{2}-1 \mid x \in \partial M, y \in \partial \mathfrak{B}_{t}\right\}, \quad t \geqslant 0 .
$$

Обобщая конструкцию (2.14), определим функцию

$$
\Omega_{M}(t)=\sup _{a \in M} \sup _{\substack{L \in \operatorname{Lin}_{2}: \\ w_{L}(L \cap(M-a)) \leqslant t}} \operatorname{diam}(L \cap(M-a)), \quad t \geqslant 0 .
$$

Также рассмотрим следующий модуль, характеризующий квазишар $M$ :

$$
\begin{aligned}
\Phi_{M}(t)=\sup \{\|x\| \mid x, y, z \in E: z+x \in \partial M, z-x \in \partial M, & \\
z+y & \notin \operatorname{int} M, z-y \notin \operatorname{int} M,\|y\| \leqslant t\}, \quad t \geqslant 0 .
\end{aligned}
$$

Используя замечания 5.2, 5.5 и сравнивая формулы (2.4), (2.5), (2.8), (2.9), (2.14), (2.15) с формулами $(5.6),(5.8),(5.10)-(5.13)$, получаем следующее замечание.

ЗАмечАниЕ 5.9. Если в качестве квазишара $M$ взять единичный шар $\mathfrak{B}_{1}$, то модули, характеризующие квазишар $M$, совпадут с соответствующими модулями пространства $E$. Таким образом, для любых чисел $t>0$ и $d>0$ справедливы равенства

$$
\begin{gathered}
\omega_{\mathfrak{B}_{1}}(t, d, \mathfrak{F})=\omega(t, d, \mathfrak{F}), \quad \theta_{\mathfrak{B}_{1}}(t, d, \mathfrak{F})=\theta(t, d, \mathfrak{F}), \\
\delta_{\mathfrak{B}_{1}}(t)=\delta(t), \quad \varrho_{\mathfrak{B}_{1}}(t)=\varrho(t), \quad \Omega_{\mathfrak{B}_{1}}(t)=\Omega(t), \quad \Phi_{\mathfrak{B}_{1}}(t)=\Phi(t) .
\end{gathered}
$$


ЗАмЕчАниЕ 5.10. Если $M=\mathfrak{B}_{1}$, квазишар $M^{\prime}$ является единичным шаром нормы $n(\cdot)$ такой, что $D(n(\cdot),\|\cdot\|) \leqslant t<1$, то согласно определению (2.6) для любого $x \in E$ справедливы неравенства $(1-t)\|x\| \leqslant n(x) \leqslant(1+t)\|x\|$. Поэтому $\frac{1}{1+t} M \subset M^{\prime} \subset \frac{1}{1-t} M$. Используя определение (5.7), получаем неравенство $h_{M}\left(M^{\prime}, M\right) \leqslant \frac{t}{1-t}$. Сравнивая формулы $(2.7)$ и $(5.9)$, имеем

$$
\nu(t, d, \mathfrak{F}) \leqslant \nu_{\mathfrak{B}_{1}}\left(\frac{t}{1-t}, d, \mathfrak{F}\right) \quad \forall t \in(0,1), \quad \forall d>0 .
$$

\section{§ 6. Оценки модулей непрерывности $M$-проекции}

ТеОрема 6.1. Пустъ строго выпуклый квазишар $M \subset$ E удовлетворяет включениям $\mathfrak{B}_{\sigma} \subset M \subset \mathfrak{B}_{\varkappa \sigma}$ при некоторъх $\sigma, \varkappa \in(0,+\infty)$. Тогда для любъх $d \in(0,1), t \in(0, \sigma d)$ справедливы неравенства

$$
\begin{aligned}
d \Phi_{M}\left(\frac{t}{8 d}\right) & -\frac{9}{8} t \leqslant \omega_{M}(t, d, \text { Conv }) \\
& \leqslant \omega_{M}(t, d, \mathcal{W C}(M)) \leqslant 2 d \Phi_{M}\left(\frac{(1+\varkappa) t}{2 \min \{d, 1-d\}}\right)+(1+\varkappa) t .
\end{aligned}
$$

СлеДСТвИЕ 6.1. Для любого строго выпуклого квазишара $M$ и любого числа $d \in(0,1)$ имеют место соотношения

$$
\omega_{M}(t, d, \mathcal{W C}(M)) \asymp \omega_{M}(t, d, \text { Conv }) \asymp \Phi_{M}(t), \quad t \rightarrow+0 .
$$

Лемма 6.1. Для любого строго выпуклого квазишара $M \subset E$, удовлетворяющего включениям $\mathfrak{B}_{\sigma} \subset M \subset \mathfrak{B}_{\varkappa \sigma}$, справедливы оченки

$$
\frac{1}{2} \Omega_{M}(t) \leqslant \Phi_{M}(t) \leqslant \Omega_{M}(8(1+\varkappa) t)+3(2 \varkappa+1) t \quad \forall t \in\left(0, \frac{\sigma}{8}\right),
$$

a значит,

$$
\Phi_{M}(t) \asymp \Omega_{M}(t), \quad t \rightarrow+0 .
$$

Лемма 6.2. Для любого равномерно выпуклого квазишара $M \subset E$ такого, что $\mathfrak{B}_{\sigma} \subset M$, справедлива оченка

$$
2 \Phi_{M}(t) \leqslant \delta_{M}^{-1}\left(\varrho_{M}(2 t)\right) \quad \forall t \in\left(0, \frac{\sigma}{2}\right] .
$$

Теорема 6.2. Пусть равномерно выпуклый квазишар $M \subset E$ удовлетворяет включениям $\mathfrak{B}_{\sigma} \subset M \subset \mathfrak{B}_{\varkappa \sigma}$ при некоторьх $\sigma, \varkappa \in(0,+\infty)$. Тогда для любых чисел $d \in(0,1)$ и $t \in(0, d)$ справедливы неравенства

$$
\begin{aligned}
\frac{d}{2} \delta_{M}^{-1}\left(\frac{t}{9 \varkappa d}\right)-\frac{3}{16} \sigma t & \leqslant \theta_{M}(t, d, \text { Conv }) \\
& \leqslant \theta_{M}(t, d, \mathcal{W C}(M)) \leqslant d \delta_{M}^{-1}\left(\frac{t}{\min \{d, 1-d\}}\right)+\varkappa \sigma t .
\end{aligned}
$$

СлеДСтвиЕ 6.2. Пусть $M \subset E$ - равномерно выпуклый ограниченный квазишар. Тогда для любого числа $d \in(0,1)$ справедливы соотношения

$$
\theta_{M}(t, d, \mathrm{Conv}) \asymp \theta_{M}(t, d, \mathcal{W C}(M)) \asymp \delta_{M}^{-1}(t), \quad t \rightarrow+0 .
$$


Теорема 6.3. Пусть равномерно выпуклый квазишар $M \subset E$ удовлетворяет включению $M \subset \mathfrak{B}_{R}$. Тогда для любых чисел $d \in(0,1)$ u $t \in(0,1)$ справедливы неравенства

$$
\begin{aligned}
& \frac{d}{2} \delta_{M}^{-1}(t)-\frac{R d t}{1-t} \leqslant \nu_{M}(t, d, \text { Conv }) \\
& \quad \leqslant \nu_{M}(t, d, \mathcal{W C}(M)) \leqslant d \delta_{M}^{-1}\left(\frac{d t}{\min \{d,(1-t)(1-d)\}}\right)+\frac{2 R d t}{1-t} .
\end{aligned}
$$

СледСТвИЕ 6.3. Пусть $M \subset E$ - равномерно выпуклый ограниченный квазишар. Тогда для любого числа $d \in(0,1)$ справедливы соотношения

$$
\nu_{M}(t, d, \mathrm{Conv}) \asymp \nu_{M}(t, d, \mathcal{W C}(M)) \asymp \delta_{M}^{-1}(t), \quad t \rightarrow+0 .
$$

\section{§ 7. Вспомогательные результаты}

Лемма 7.1. Пусть $M-$ квазишар, $A \in \mathcal{W C}(M)$, векторь $x_{1}, x_{2} \in E$ удовлетворяют условиям $d_{M}\left(x_{1}, A\right)=d_{M}\left(x_{2}, A\right)=d \in(0,1)$ u $a_{i} \in P_{M}\left(x_{i}, A\right)$ nри $i=1,2$. Тогда для векторов $z_{i}=\frac{x_{i}-a_{i}}{d}$ справедливо неравенство

$$
\left\|z_{1}-z_{2}\right\| \leqslant 2 \Phi_{M}\left(\frac{\left\|x_{1}-x_{2}\right\|}{2 \min \{d, 1-d\}}\right) .
$$

ДоказАтельство. Из условия $a_{i} \in P_{M}\left(x_{i}, A\right)$ согласно формуле (5.3) следуют равенства $\mu_{M}\left(x_{i}-a_{i}\right)=d_{M}\left(x_{i}, A\right)=d$. Поэтому в силу равенства $(5.4)$ имеем $z_{i} \in N_{M}\left(a_{i}, A\right), \mu_{M}\left(z_{i}\right)=1$ при $i=1,2$. Обозначим

$$
z=\frac{z_{1}+z_{2}}{2}, \quad y=\frac{x_{1}-x_{2}}{2 \min \{d, 1-d\}} .
$$

Покажем, что

$$
\mu_{M}(z+y) \geqslant 1
$$

Из включения $A \in \mathcal{W C}(M)$ и формулы (5.5) следует, что $a_{i} \in P_{M}\left(a_{i}+z_{i}, A\right)$, а значит, $d_{M}\left(a_{i}+z_{i}, A\right)=\mu_{M}\left(z_{i}\right)=1$ при $i=1,2$. Учитывая включения $a_{1}, a_{2} \in A$, получаем

$$
\mu_{M}\left(a_{1}+z_{1}-a_{2}\right) \geqslant 1, \quad \mu_{M}\left(a_{2}+z_{2}-a_{1}\right) \geqslant 1 .
$$

Рассмотрим случай $d \in\left[\frac{1}{2}, 1\right)$. Тогда

$$
y=\frac{x_{1}-x_{2}}{2(1-d)}, \quad a_{1}+z_{1}-a_{2}=(2 d-1) z_{2}+2(1-d)(z+y) .
$$

Согласно первому из неравенств в (7.3) имеем

$1 \leqslant \mu_{M}\left(a_{1}+z_{1}-a_{2}\right) \leqslant(2 d-1) \mu_{M}\left(z_{2}\right)+2(1-d) \mu_{M}(z+y)=2 d-1+2(1-d) \mu_{M}(z+y)$, откуда следует неравенство (7.2).

Пусть теперь $d \in\left(0, \frac{1}{2}\right)$. В этом случае

$$
y=\frac{x_{1}-x_{2}}{2 d}, \quad a_{1}+z_{1}-a_{2}=(1-2 d) z_{1}+2(z+y) d .
$$


Поэтому

$$
1 \leqslant \mu_{M}\left(a_{1}+z_{1}-a_{2}\right) \leqslant(1-2 d) \mu_{M}\left(z_{1}\right)+2 d \mu_{M}(z+y)=1-2 d+2 d \mu_{M}(z+y) .
$$

Таким образом, неравенство (7.2) доказано. Аналогично, используя второе из неравенств $(7.3)$, получаем $\mu_{M}(z-y) \geqslant 1$. Следовательно, $z+y \notin \operatorname{int} M$ и $z-y \notin$ int $M$. Используя включения $z_{i} \in \partial M$ при $i=1,2$, согласно равенству (5.13) приходим к неравенству $\Phi_{M}(\|y\|) \geqslant \frac{\left\|z_{1}-z_{2}\right\|}{2}$.

Лемма 7.2. Пусть квазишар $M \subset E$ удовлетворяет включениям $\mathfrak{B}_{\sigma} \subset$ $M \subset \mathfrak{B}_{\varkappa \sigma}$. Тогда для любых $t>0, d_{1} \in(0,1)$ справедливо неравенство

$$
\omega_{M}\left(t, d_{1}, \mathcal{W C}(M)\right) \leqslant 2 d_{1} \Phi\left(\frac{(1+\varkappa) t}{2 \min \left\{d_{1}, 1-d_{1}\right\}}\right)+(1+\varkappa) t .
$$

ДокАЗАТЕЛьство. Зафиксируем произвольные числа $t>0, d_{1} \in(0,1)$. Пусть

$$
\begin{gathered}
A \in \mathcal{W C}(M), \quad x_{1}, x_{2} \in E, \quad\left\|x_{1}-x_{2}\right\| \leqslant t, \\
d_{M}\left(x_{1}, A\right)=d_{1}, \quad a_{1} \in P_{M}\left(x_{1}, A\right), \quad a_{2} \in P_{M}\left(x_{2}, A\right) .
\end{gathered}
$$

Покажем, что

$$
\left\|a_{1}-a_{2}\right\| \leqslant 2 d_{1} \Phi_{M}\left(\frac{(1+\varkappa) t}{2 \min \left\{d_{1}, 1-d_{1}\right\}}\right)+(1+\varkappa) t .
$$

Обозначим $d_{2}=d_{M}\left(x_{2}, A\right)$. Используя замечание 5.3 , имеем

$$
\left|d_{1}-d_{2}\right| \leqslant \frac{\left\|x_{1}-x_{2}\right\|}{\sigma} \leqslant \frac{t}{\sigma}
$$

Рассмотрим случай $d_{2}=0$. Тогда $a_{2}=x_{2}$. Поскольку $\mu_{M}\left(x_{1}-a_{1}\right)=d_{1}$ и $M \subset \mathfrak{B}_{\varkappa \sigma}$, то $\left\|a_{1}-x_{1}\right\| \leqslant d_{1} \varkappa \sigma$. Используя неравенства $(7.5)$, имеем $d_{1} \leqslant \frac{t}{\sigma}$. Следовательно,

$$
\left\|a_{1}-a_{2}\right\|=\left\|a_{1}-x_{2}\right\| \leqslant\left\|x_{1}-x_{2}\right\|+\left\|a_{1}-x_{1}\right\| \leqslant t+\varkappa t .
$$

Таким образом, в данном случае неравенство (7.4) выполнено.

Пусть теперь $d_{2}>0$. Обозначим

$$
z_{1}=\frac{x_{1}-a_{1}}{d_{1}}, \quad z_{2}=\frac{x_{2}-a_{2}}{d_{2}}, \quad x_{2}^{\prime}=a_{2}+d_{1} z_{2} .
$$

Поскольку $A \in \mathcal{W C}(M)$ и $d_{1} \in(0,1)$, то $a_{2} \in P_{M}\left(x_{2}^{\prime}, A\right)$ и $d_{M}\left(x_{2}^{\prime}, A\right)=d_{1}$. В силу леммы 7.1 имеем

$$
\left\|z_{1}-z_{2}\right\| \leqslant 2 \Phi_{M}\left(\frac{\left\|x_{1}-x_{2}^{\prime}\right\|}{2 \min \left\{d_{1}, 1-d_{1}\right\}}\right)
$$

Замечая, что

$$
\begin{aligned}
&\left\|a_{1}-a_{2}\right\|=\left\|x_{1}-x_{2}-\left(d_{1} z_{1}-d_{2} z_{2}\right)\right\| \\
& \leqslant\left\|x_{1}-x_{2}\right\|+\varkappa \sigma\left|d_{1}-d_{2}\right|+d_{1}\left\|z_{1}-z_{2}\right\| \leqslant d_{1}\left\|z_{1}-z_{2}\right\|+(1+\varkappa) t, \\
&\left\|x_{1}-x_{2}^{\prime}\right\| \leqslant\left\|x_{1}-x_{2}\right\|+\left\|x_{2}-x_{2}^{\prime}\right\| \leqslant t+\left\|z_{2}\right\|\left|d_{1}-d_{2}\right| \leqslant(1+\varkappa) t,
\end{aligned}
$$

получаем неравенство (7.4). Согласно определению (5.6) модуля $\omega_{M}$ неравенство (7.4) влечет доказываемое утверждение. 
ЛЕмма 7.3. Пусть $M$ - ограниченный и строго выпуклый квазишар, $\sigma>0$, $\mathfrak{B}_{\sigma} \subset M$ и вектори $x, y, z \in E$ удовлетворяют условиям $z+x \in M, z-x \in M$, $z+y \notin \operatorname{int} M$. Пусть прямая $\ell=\{u(\tau) \mid \tau \in \mathbb{R}\}$ и отрезок $A=[-z+x,-z-x-$ $6 y]=\{u(\tau) \mid \tau \in[0,2]\}$ параметризованы функиией $u(\tau)=-z+x-\tau(x+3 y)$. Пусть

$$
P_{M}(0, \ell)=\left\{u\left(\tau_{0}\right)\right\}, \quad P_{M}(0, A)=\left\{u\left(\tau_{1}\right)\right\} .
$$

Тогда

$$
\begin{gathered}
\tau_{0} \leqslant \frac{1}{2}, \\
0 \leqslant \tau_{1} \leqslant \frac{1}{2}, \\
1-\frac{2\|y\|}{\sigma} \leqslant d_{M}(0, A) \leqslant 1 .
\end{gathered}
$$

Если дополнительно $z-y \notin \operatorname{int} M$, mо

$$
1-\frac{2\|y\|}{\sigma} \leqslant d_{M}(0, \ell) \leqslant 1
$$

ДоказАтельство. Из соотношений $-z+x \in \ell \cap A \cap(-M)$ и (7.6) следует, что

$$
\begin{aligned}
& \mu_{M}\left(-u\left(\tau_{0}\right)\right)=d_{M}(0, \ell) \leqslant \mu_{M}(z-x) \leqslant 1, \\
& \mu_{M}\left(-u\left(\tau_{1}\right)\right)=d_{M}(0, A) \leqslant \mu_{M}(z-x) \leqslant 1,
\end{aligned}
$$

а значит, $-u\left(\tau_{i}\right) \in M$ при $i=0,1$. Предположим, что неравенство (7.7) не выполнено: $\tau_{0}>\frac{1}{2}$. В силу строгой выпуклости квазишара $M$ и соотношений

$$
z-x+\frac{1}{2}(x+3 y)=-u\left(\frac{1}{2}\right) \in\left(-u(0),-u\left(\tau_{0}\right)\right)=\left(z-x,-u\left(\tau_{0}\right)\right)
$$

получаем включение $z-x+\frac{1}{2}(x+3 y) \in \operatorname{int} M$, которое с учетом равенства $z+y=\frac{2}{3}\left(z-x+\frac{1}{2}(x+3 y)\right)+\frac{1}{3}(z+x)$ и включения $z+x \in M$ противоречит условию $z+y \notin \operatorname{int} M$. Таким образом, неравенство (7.7) доказано.

Поскольку $u\left(\tau_{1}\right) \in A$, то $\tau_{1} \geqslant 0$. Предположим, что $\tau_{1}>\frac{1}{2}$. Тогда $u\left(\tau_{1}\right) \neq u\left(\tau_{0}\right)$. Из соотношений $u\left(\tau_{1}\right) \in A \subset \ell, P_{M}(0, \ell)=\left\{u\left(\tau_{0}\right)\right\}$ следует, что $\mu_{M}\left(-u\left(\tau_{1}\right)\right)>d_{M}(0, \ell)=\mu_{M}\left(-u\left(\tau_{0}\right)\right)$. Используя включение $u\left(\frac{1}{2}\right) \in$ $\left[u\left(\tau_{0}\right), u\left(\tau_{1}\right)\right)$, получаем неравенство $\mu_{M}\left(-u\left(\frac{1}{2}\right)\right)<\mu_{M}\left(-u\left(\tau_{1}\right)\right)$. Согласно включению $u\left(\frac{1}{2}\right) \in A$ имеем $d_{M}(0, A) \leqslant \mu_{M}\left(-u\left(\frac{1}{2}\right)\right)<\mu_{M}\left(-u\left(\tau_{1}\right)\right)$. Это противоречит равенству $P_{M}(0, A)=\left\{u\left(\tau_{1}\right)\right\}$. Таким образом, неравенства $(7.8)$ также доказаны.

При $i \in\{0,1\}$ обозначим

$$
\begin{gathered}
\lambda_{i}=\frac{1-\tau_{i}}{2-\tau_{i}}, \\
v_{i}^{+}=\left(1-\lambda_{i}\right)\left(2 y-u\left(\tau_{i}\right)\right)+\lambda_{i}(z+x), \quad v_{i}^{-}=\left(1-\lambda_{i}\right)\left(-2 y-u\left(\tau_{i}\right)\right)+\lambda_{i}(z+x) .
\end{gathered}
$$

Тогда

$$
\lambda_{i} \in(0,1), \quad v_{i}^{+}=z+\frac{3 \tau_{i}+2}{2-\tau_{i}} y, \quad v_{i}^{-}=z+\frac{3 \tau_{i}-2}{2-\tau_{i}} y
$$


Зафиксируем индекс $i \in\{0,1\}$. Если $\tau_{i} \in\left[0, \frac{1}{2}\right]$, то $\frac{3 \tau_{i}+2}{2-\tau_{i}} \geqslant 1$. Поэтому в силу (7.13) получаем включение $z+y \in\left[z, v_{i}^{+}\right]$. Используя включения $z \in \operatorname{int} M$ и $z+y \notin \operatorname{int} M$, приходим к соотношению $v_{i}^{+} \notin \operatorname{int} M$. Поэтому $2 y-u\left(\tau_{i}\right) \notin \operatorname{int} M$. Итак,

$$
\tau_{i} \in\left[0, \frac{1}{2}\right] \quad \Longrightarrow \quad \mu_{M}\left(2 y-u\left(\tau_{i}\right)\right) \geqslant 1 \quad \forall i \in\{0,1\} .
$$

В силу неравенств $(7.8)$ имеем $\mu_{M}\left(2 y-u\left(\tau_{1}\right)\right) \geqslant 1$. Следовательно, согласно соотношениям (7.12) и замечанию 5.3 справедливы соотношения

$$
1 \geqslant d_{M}(0, A)=\mu_{M}\left(-u\left(\tau_{1}\right)\right) \geqslant \mu_{M}\left(2 y-u\left(\tau_{1}\right)\right)-\frac{2\|y\|}{\sigma} \geqslant 1-\frac{2\|y\|}{\sigma} .
$$

Таким образом, доказаны неравенства (7.9). Пусть теперь $z-y \notin \operatorname{int} M$. Если $\tau_{0} \geqslant 0$, то аналогичными рассуждениями получаем неравенства (7.10). Теперь рассмотрим случай $\tau_{0}<0$. В этом случае из соотношений $\frac{3 \tau_{0}-2}{2-\tau_{0}}<-1$ и $(7.13)$ следует, что $z-y \in\left[z, v_{0}^{-}\right]$. Учитывая соотношения $z \in \operatorname{int} M$ и $z-y \notin \operatorname{int} M$, имеем $v_{0}^{-} \notin \operatorname{int} M$. Поэтому $-2 y-u\left(\tau_{0}\right) \notin \operatorname{int} M$, т. е. $\mu_{M}\left(-2 y-u\left(\tau_{0}\right)\right) \geqslant 1$. Согласно замечанию 5.3 и соотношениям (7.11) получаем неравенства (7.10).

Лемма 7.4. Пусть $M$ - ограниченный и строго выпуклый квазишар, $\sigma>0$, $\mathfrak{B}_{\sigma} \subset M$. Тогда для любых чисел $t \in\left(0, \frac{\sigma}{8}\right)$ u $\varepsilon>0$ в пространстве $E$ существуют прямая $\ell$ и вектор $y \in \mathfrak{B}_{t}$ такие, что

$$
\begin{gathered}
P_{M}(0, \ell-3 y)=\left\{x_{1}\right\}, \quad P_{M}(0, \ell+3 y)=\left\{x_{2}\right\}, \\
\left\|x_{1}-x_{2}\right\| \geqslant \Phi_{M}(t)-3 t-\varepsilon \\
d_{M}(0, \ell-3 y) \in\left[\frac{3}{4}, 1\right] .
\end{gathered}
$$

ДокАЗАтЕльство. Зафиксируем произвольные $t \in\left(0, \frac{\sigma}{8}\right)$ и $\varepsilon>0$. В силу определения (5.13) модуля $\Phi_{M}$ существуют векторы $x, y, z \in E$ такие, что

$$
\begin{gathered}
z+x \in \partial M, \quad z-x \in \partial M, \quad z+y \notin \operatorname{int} M, \quad z-y \notin \operatorname{int} M, \\
\|y\| \leqslant t, \quad\|x\|>\Phi_{M}(t)-\varepsilon .
\end{gathered}
$$

Определим прямую

$$
\ell=\{-z-t(x+3 y) \mid t \in \mathbb{R}\} .
$$

Заметим, что для прямых $\ell^{-}=\ell-3 y, \ell^{+}=\ell+3 y$ и функций

$$
u^{-}(\tau)=-z+x-\tau(x+3 y), \quad u^{+}(\tau)=-z-x+\tau(x+3 y)
$$

справедливы равенства

$$
\ell^{-}=\left\{u^{-}(\tau) \mid \tau \in \mathbb{R}\right\}, \quad \ell^{+}=\left\{u^{+}(\tau) \mid \tau \in \mathbb{R}\right\} .
$$

В силу строгой выпуклости квазишара $M$ множества $P_{M}\left(0, \ell^{-}\right)$и $P_{M}\left(0, \ell^{+}\right)$одноэлементны. Поэтому при некоторых $x_{1}, x_{2} \in E$ справедливы равенства (7.15). Согласно уравнениям (7.19) найдутся числа $t_{1}, t_{2}$ такие, что $x_{1}=u^{-}\left(t_{1}\right)$, $x_{2}=u^{+}\left(t_{2}\right)$, т. е.

$$
x_{1}=-z+x-t_{1}(x+3 y), \quad x_{2}=-z-x+t_{2}(x+3 y) .
$$


Применяя лемму 7.3 для прямой $\ell^{-}$и замечая, что $\|y\| \leqslant t<\frac{\sigma}{8}$, имеем $t_{1} \leqslant \frac{1}{2}$ и

$$
\frac{3}{4} \leqslant 1-\frac{2\|y\|}{\sigma} \leqslant d_{M}\left(0, \ell^{-}\right) \leqslant 1 .
$$

Применение леммы 7.3 для прямой $\ell^{+}$и векторов $-x,-y$ вместо векторов $x, y$ дает неравенство $t_{2} \leqslant \frac{1}{2}$. Из равенств (7.20) получаем

$\left\|x_{1}-x_{2}\right\|=\left\|2 x-\left(t_{1}+t_{2}\right)(x+3 y)\right\| \geqslant\left(2-t_{1}-t_{2}\right)\|x\|-3\left|t_{1}+t_{2}\right|\|y\|, \quad t_{1}+t_{2} \leqslant 1$.

Покажем, что

$$
\left\|x_{1}-x_{2}\right\| \geqslant\|x\|-3\|y\| .
$$

Если $\|x\| \leqslant 3\|y\|$, то неравенство (7.23) выполнено. Поэтому будем предполагать, что $\|x\|>3\|y\|$. Обозначим $T=t_{1}+t_{2}$. Тогда в силу (7.22) имеем

$$
T \leqslant 1 \quad \text { и } \quad\left\|x_{1}-x_{2}\right\| \geqslant(2-T)\|x\|-3|T|\|y\| \geqslant\|x\|-3\|y\| .
$$

Последнее неравенство следует из того, что

$$
(1-T)\|x\| \geqslant 3(1-T)\|y\| \geqslant 3(|T|-1)\|y\| .
$$

Итак, неравенство (7.23) доказано. Используя неравенства (7.18), (7.23), получаем

$$
\left\|x_{1}-x_{2}\right\| \geqslant\|x\|-3\|y\| \geqslant\|x\|-3 t>\Phi_{M}(t)-3 t-\varepsilon .
$$

Таким образом, неравенство (7.16) доказано. Включение (7.17) следует из неравенств (7.21).

Пусть заданы семейство $\mathfrak{F}$ подмножеств пространства $E$ и число $r>0$. Через $r \mathfrak{F}$ обозначим семейство множеств $r A$, где $A \in \mathfrak{F}$.

ЗАмЕчАниЕ 7.1. Из определений (5.6), (5.8), (5.9) следует, что для любого квазишара $M$, любых положительных чисел $t, d, r$ и любого семейства $\mathfrak{F}$ подмножеств пространства $E$ справедливы равенства

$$
\begin{gathered}
\omega_{M}(r t, r d, r \mathfrak{F})=r \omega_{M}(t, d, \mathfrak{F}), \quad \theta_{M}(r t, r d, r \mathfrak{F})=r \theta_{M}(t, d, \mathfrak{F}), \\
\nu_{M}(t, r d, r \mathfrak{F})=r \nu_{M}(t, d, \mathfrak{F}) .
\end{gathered}
$$

В частности, используя равенства $r$ Conv $=$ Conv, $r \mathcal{P} \mathcal{R}(1)=\mathcal{P} \mathcal{R}(r)$, для любых положительных чисел $t, d, r$ получаем равенства

$$
\begin{aligned}
\omega_{M}(r t, r d, \mathcal{P} \mathcal{R}(r))=r \omega_{M}(t, d, \mathcal{P} \mathcal{R}(1)), & \omega_{M}(r t, r d, \text { Conv })=r \omega_{M}(t, d, \text { Conv }), \\
\theta_{M}(r t, r d, \mathcal{P} \mathcal{R}(r))=r \theta_{M}(t, d, \mathcal{P} \mathcal{R}(1)), & \theta_{M}(r t, r d, \text { Conv })=r \theta_{M}(t, d, \text { Conv }), \\
\nu_{M}(t, r d, \mathcal{P} \mathcal{R}(r))=r \nu_{M}(t, d, \mathcal{P} \mathcal{R}(1)), & \nu_{M}(t, r d, \mathrm{Conv})=r \nu_{M}(t, d, \text { Conv }) .
\end{aligned}
$$

Лемма 7.5. Пусть строго выпуклый квазишар $M \subset E$ удовлетворяет включениям $\mathfrak{B}_{\sigma} \subset M \subset \mathfrak{B}_{\varkappa \sigma}$ при некоторых $\sigma, \varkappa \in(0,+\infty)$. Тогда для любых $d>0, t \in(0, \sigma d)$ справедливо неравенство

$$
d \cdot \Phi_{M}\left(\frac{t}{8 d}\right)-\frac{9}{8} t \leqslant \omega_{M}(t, d, \text { Conv }) .
$$


ДокАЗАТЕльство. Зафиксируем произвольные числа $d>0, t \in(0, \sigma d), \varepsilon>0$ и определим число $\tau=\frac{t}{8 d}$. Тогда $\tau \in\left(0, \frac{\sigma}{8}\right)$. В силу леммы 7.4 существуют прямая $\ell$ и вектор $y \in \mathfrak{B}_{\tau}$ такие, что

$$
\begin{gathered}
P_{M}(0, \ell-3 y)=\left\{x_{1}\right\}, \quad P_{M}(0, \ell+3 y)=\left\{x_{2}\right\}, \\
\left\|x_{1}-x_{2}\right\| \geqslant \Phi_{M}(\tau)-3 \tau-\varepsilon, \quad d_{M}(0, \ell-3 y) \in\left[\frac{3}{4}, 1\right] .
\end{gathered}
$$

Используя первое из равенств (7.27), получаем

$$
P_{M}(6 y, \ell+3 y)=6 y+P_{M}(0, \ell-3 y)=\left\{x_{1}+6 y\right\} .
$$

Обозначим $d_{1}=d_{M}(0, \ell-3 y)$. Тогда $\frac{3}{4} \leqslant d_{1} \leqslant 1$. Принимая во внимание второе из равенств (7.27), по определению (5.6) модуля $\omega_{M}$ получаем $\omega_{M}\left(6 \tau, d_{1}\right.$, Conv $) \geqslant\left\|x_{1}+6 y-x_{2}\right\|$. Учитывая соотношения $t=8 \tau d \geqslant \frac{6 \tau d}{d_{1}}$ и второе из равенств (7.24), имеем

$$
\begin{aligned}
\omega_{M}(t, d, \text { Conv }) & \geqslant \omega_{M}\left(\frac{6 \tau d}{d_{1}}, d, \text { Conv }\right)=\frac{d}{d_{1}} \omega_{M}\left(6 \tau, d_{1}, \text { Conv }\right) \\
& \geqslant d \cdot \omega_{M}\left(6 \tau, d_{1}, \text { Conv }\right) \geqslant d\left\|x_{1}+6 y-x_{2}\right\| \geqslant d\left\|x_{1}-x_{2}\right\|-6\|y\| d \\
& \geqslant d\left(\Phi_{M}(\tau)-3 \tau-\varepsilon\right)-6 \tau d=d \Phi_{M}(\tau)-\frac{9}{8} t-\varepsilon d .
\end{aligned}
$$

Переходя к пределу при $\varepsilon \rightarrow+0$, получаем доказываемое неравенство.

Лемма 7.6. Пусть строго выпуклый квазишар $M \subset E$ удовлетворяет включениям $\mathfrak{B}_{\sigma} \subset M \subset \mathfrak{B}_{\varkappa \sigma}$ при некоторых $\sigma, \varkappa \in(0,+\infty)$. Тогда

$$
\Phi_{M}(t) \leqslant \Omega_{M}(8(1+\varkappa) t)+6 \varkappa t+3 t \quad \forall t \in\left(0, \frac{\sigma}{8}\right) .
$$

ДокАЗАТЕЛЬство. Зафиксируем произвольные числа $t \in\left(0, \frac{\sigma}{8}\right)$ и $\varepsilon>0$ и покажем, что

$$
\Phi_{M}(t) \leqslant \Omega_{M}(8(1+\varkappa) t)+6 \varkappa t+3 t+\varepsilon .
$$

В силу леммы 7.4 существуют прямая $\ell$ и вектор $y \in \mathfrak{B}_{t}$ такие, что прямые $\ell_{1}=\ell-3 y$ и $\ell_{2}=\ell+3 y$ удовлетворяют соотношениям

$$
\begin{gathered}
P_{M}\left(0, \ell_{i}\right)=\left\{x_{i}\right\} \quad \forall i \in\{1,2\} \\
\left\|x_{1}-x_{2}\right\| \geqslant \Phi_{M}(t)-3 t-\varepsilon \\
d_{M}\left(0, \ell_{1}\right) \in\left[\frac{3}{4}, 1\right] .
\end{gathered}
$$

При $i \in\{1,2\}$ обозначим

$$
d_{i}=d_{M}\left(0, \ell_{i}\right), \quad \ell_{i}^{\prime}=\frac{1}{d_{i}} \ell_{i}, \quad x_{i}^{\prime}=\frac{1}{d_{i}} x_{i} .
$$

Поскольку $d_{1}=d_{M}\left(0, \ell_{1}\right)=d_{M}(3 y, \ell), d_{2}=d_{M}\left(0, \ell_{2}\right)=d_{M}(-3 y, \ell)$, то согласно замечанию 5.3 имеем

$$
\left|d_{1}-d_{2}\right| \leqslant \frac{6\|y\|}{\sigma} \leqslant \frac{6 t}{\sigma} .
$$


Используя равенства $\mu_{M}\left(-x_{2}\right)=d_{M}\left(0, \ell_{2}\right)=d_{2}$, имеем $x_{2}^{\prime}=\frac{x_{2}}{d_{2}} \in-M \subset \mathfrak{B}_{\varkappa \sigma}$. Следовательно,

$$
\begin{aligned}
\left|d_{1}-d_{2}\right| \cdot & \left\|x_{2}^{\prime}\right\| \leqslant 6 \varkappa t \\
\left\|x_{1}-x_{2}\right\|=\left\|d_{1} x_{1}^{\prime}-d_{2} x_{2}^{\prime}\right\| & \leqslant\left|d_{1}-d_{2}\right|\left\|x_{2}^{\prime}\right\|+d_{1}\left\|x_{1}^{\prime}-x_{2}^{\prime}\right\| \\
& \leqslant 6 \varkappa t+\left\|x_{1}^{\prime}-x_{2}^{\prime}\right\| .
\end{aligned}
$$

Если $\left\|x_{1}-x_{2}\right\| \leqslant 6 x t$, то неравенство (7.28) следует из неравенства (7.30). Поэтому будем предполагать, что $\left\|x_{1}-x_{2}\right\|>6 \varkappa t$. Используя неравенства (7.34), имеем $x_{1}^{\prime} \neq x_{2}^{\prime}$. Поскольку прямая $\ell_{0}=\ell_{2}-x_{2}$ проходит через начало координат и $\ell_{2}=\ell_{0}+x_{2}, \ell_{1}=\ell_{0}+x_{2}-6 y$, то согласно равенствам (7.32) имеем

$$
\ell_{1}^{\prime}=\ell_{0}+\frac{x_{2}-6 y}{d_{1}}, \quad \ell_{2}^{\prime}=\ell_{0}+\frac{x_{2}}{d_{2}} .
$$

В силу соотношений (7.29), (7.32) получаем

$$
P_{M}\left(0, \ell_{i}^{\prime}\right)=\left\{x_{i}^{\prime}\right\}, \quad d_{M}\left(0, \ell_{i}^{\prime}\right)=1, \quad i=1,2 .
$$

Поскольку $x_{1}^{\prime} \neq x_{2}^{\prime}$, то $\ell_{1}^{\prime} \neq \ell_{2}^{\prime}$ и $x_{1}^{\prime} \notin \ell_{2}^{\prime}$. Пусть $L \in \operatorname{Lin}_{2}-$ двумерное линейное подпространство, содержащее прямую $\ell_{2}^{\prime}-x_{1}^{\prime}$. Используя параллельность прямых $\ell_{1}^{\prime}$ и $\ell_{2}^{\prime}$, получаем включение $\ell_{1}^{\prime}-x_{1}^{\prime} \subset L$. Из равенств (7.36) следует, что множество $A=\left(M-x_{1}^{\prime}\right) \cap L$ заключено между параллельными прямыми $\ell_{1}^{\prime}-x_{1}^{\prime}$ и $\ell_{2}^{\prime}-x_{1}^{\prime}$. Следовательно, по определению ширины (2.13) из равенств (7.35) вытекает неравенство

$$
w_{L}(A) \leqslant\left\|\frac{x_{2}-6 y}{d_{1}}-\frac{x_{2}}{d_{2}}\right\| .
$$

Отсюда и из неравенства (7.33) и включения (7.31) получаем

$$
w_{L}(A) \leqslant \frac{\left|d_{1}-d_{2}\right|\left\|x_{2}^{\prime}\right\|+6\|y\|}{d_{1}} \leqslant \frac{4}{3}(6 \varkappa t+6 t)=8(1+\varkappa) t .
$$

Используя определение (5.12) функции $\Omega_{M}$ и неравенства (7.34), приходим к неравенствам

$$
\Omega_{M}(8(1+\varkappa) t) \geqslant \operatorname{diam} A \geqslant\left\|x_{1}^{\prime}-x_{2}^{\prime}\right\| \geqslant\left\|x_{1}-x_{2}\right\|-6 \varkappa t .
$$

Отсюда и из неравенства (7.30) следует неравенство (7.28). Переходя в неравенстве (7.28) к пределу при $\varepsilon \rightarrow+0$, получаем доказываемое неравенство.

ЛЕмма 7.7. Для любого строго выпуклого квазишара $M \subset E$ и для любого числа $t>0$ справедливо неравенство $\Omega_{M}(t) \leqslant 2 \Phi_{M}(t)$.

ДокАЗАТЕЛЬство. Зафиксируем произвольный строго выпуклый квазишар $M \subset E$ и произвольное число $t>0$. Если $\Omega_{M}(t)=0$, то доказываемое неравенство справедливо. Поэтому будем предполагать, что $\Omega_{M}(t)>0$. Зафиксируем число $\xi \in\left(0, \Omega_{M}(t)\right)$. В силу определения $(5.12)$ существуют вектор $a \in M$ и подпространство $L \in \operatorname{Lin}_{2}$ такие, что $w_{L}(L \cap(M-a)) \leqslant t$ и $\operatorname{diam}(L \cap(M-a))>\xi$. Выберем векторы $x_{1}, x_{2} \in L \cap(\partial M-a)$ из условия $\left\|x_{1}-x_{2}\right\|>\xi$. Обозначим

$$
z=\frac{x_{1}+x_{2}}{2}+a, \quad x=\frac{x_{1}-x_{2}}{2} .
$$


В силу строгой выпуклости квазишара $M$ и включений $z+x \in \partial M, z-x \in \partial M$ получаем включение $z \in \operatorname{int} M$. Поэтому существует число $\sigma \in\left(0, \frac{1}{3}\right)$ такое, что $\mathfrak{B}_{3 t \sigma}(z) \subset M$. Из неравенства $w_{L}(L \cap(M-a)) \leqslant t$ и определения ширины $(2.13)$ следует существование функционала $f \in L^{*}$ такого, что

$$
\|f\|=1, \quad \sup _{a_{1}, a_{2} \in L \cap(M-a)}\left\langle f, a_{1}-a_{2}\right\rangle \leqslant t(1+\sigma) .
$$

Выберем вектор $y \in L$ из условий $\|y\|=t,\langle f, y\rangle \geqslant\|y\|(1-\sigma)=t(1-\sigma)$. Предположим, что $z+y \in M$. Поскольку $z-3 \sigma y \in \mathfrak{B}_{3 t \sigma}(z) \subset M$, то

$t(1+\sigma) \geqslant \sup _{a_{1}, a_{2} \in L \cap(M-a)}\left\langle f, a_{1}-a_{2}\right\rangle \geqslant\langle f, y\rangle(1+3 \sigma) \geqslant t(1-\sigma)(1+3 \sigma)>t(1+\sigma)$.

Полученное противоречие показывает, что $z+y \notin M$. Аналогично, $z-y \notin M$. Используя определение (5.13) модуля $\Phi_{M}$, получаем $\Phi_{M}(t) \geqslant\|x\|>\frac{\xi}{2}$. Переходя к пределу при $\xi \rightarrow \Omega_{M}(t)$, получаем доказываемое неравенство.

Лемма 7.8. Пусть заданъь квазишар $M$, векторы $z, w \in M$ и число $\lambda \in$ $(0,1)$. Тогда

$$
2-\mu_{M}(w+z) \leqslant \frac{1-\mu_{M}(\lambda w+(1-\lambda) z)}{\min \{\lambda, 1-\lambda\}} .
$$

ДоказАтельство. Рассмотрим сначала случай $\lambda \in\left(0, \frac{1}{2}\right]$. Из равенства $\lambda w+(1-\lambda) z=\lambda(w+z)+(1-2 \lambda) z$ следует, что

$$
\mu_{M}(\lambda w+(1-\lambda) z) \leqslant \lambda \mu_{M}(w+z)+(1-2 \lambda) \mu_{M}(z) \leqslant \lambda \mu_{M}(w+z)+1-2 \lambda .
$$

Поэтому в данном случае доказываемое неравенство справедливо. Случай $\lambda \in$ $\left(\frac{1}{2}, 1\right)$ сводится к предыдущему, если выполнить замены $\lambda \leftrightarrow 1-\lambda, w \leftrightarrow z$.

ЛЕмма 7.9. Пусть $M \subset E-$ равномерно выпуклый квазишар,

$A \in \mathcal{W C}(M), \quad x \in E, \quad d_{1}=d_{M}(x, A) \in(0,1), \quad a_{1} \in P_{M}(x, A), \quad a_{2} \in E$,

$$
d_{1}^{\prime}>0, \quad \mu_{M}\left(x-a_{2}\right) \leqslant d_{1}^{\prime}, \quad w=\frac{x-a_{2}}{d_{1}^{\prime}}, \quad \tau=\frac{d_{1}^{\prime}-d_{1}+d_{M}\left(a_{2}, A\right)}{2 \min \left\{d_{1}^{\prime}, 1-d_{1}\right\}} .
$$

Тогда

$$
\left\|a_{2}-a_{1}\right\| \leqslant d_{1} \delta_{M}^{-1}(\tau)+\left|d_{1}^{\prime}-d_{1}\right|\|w\| .
$$

ДоказАтельство. Обозначим $z=\frac{x-a_{1}}{d_{1}}$. Поскольку $d_{1}=d_{M}(x, A)$ и $a_{1} \in$ $P_{M}(x, A)$, то $\mu_{M}(z)=1$ и по определению (5.4) конуса $M$-нормалей имеем $z \in N_{M}\left(a_{1}, A\right)$. Используя условие $A \in \mathcal{W C}(M)$, в силу соотношения (5.5) получаем равенство $d_{M}\left(a_{1}+z, A\right)=1$. Следовательно,

$$
1=d_{M}\left(a_{1}+z, A\right) \leqslant d_{M}\left(a_{2}, A\right)+\mu_{M}\left(a_{1}+z-a_{2}\right) .
$$

Замечая, что $a_{1}+z-a_{2}=\left(1-d_{1}\right) z+d_{1}^{\prime} w$, и полагая $\lambda=\frac{d_{1}^{\prime}}{1-d_{1}+d_{1}^{\prime}}$, имеем

$$
\mu_{M}((1-\lambda) z+\lambda w) \geqslant \frac{1-d_{M}\left(a_{2}, A\right)}{1-d_{1}+d_{1}^{\prime}}
$$


Из леммы 7.8 следует, что

$$
2-\mu_{M}(w+z) \leqslant \frac{1}{\min \{\lambda, 1-\lambda\}}\left(1-\frac{1-d_{M}\left(a_{2}, A\right)}{1-d_{1}+d_{1}^{\prime}}\right)=2 \tau .
$$

Поэтому, учитывая включения $z \in \partial M, w \in M$ и определение модуля выпуклости (5.10), приходим к неравенству

$$
\|w-z\| \leqslant \delta_{M}^{-1}(\tau) .
$$

Поскольку $a_{1}=x-d_{1} z, a_{2}=x-d_{1}^{\prime} w$, то $a_{1}-a_{2}=d_{1}(w-z)+\left(d_{1}^{\prime}-d_{1}\right) w$. Используя неравенство (7.37), получаем доказываемое утверждение.

Отклонением множества $A \subset E$ от множества $B \subset E$ относителъно квазишара $M$ называется

$$
h_{M}^{+}(A, B)=\sup _{a \in A} d_{M}(a, B) .
$$

Лемма 7.10. Пусть $M_{1}$ - равномерно выпуклый квазишар в $E, M_{2}$ - произвольный квазишар в E, и пусть

$$
\begin{gathered}
A_{1} \in \mathcal{W C}\left(M_{1}\right), \quad A_{2} \subset E, \quad x \in E, \quad d_{1}=d_{M_{1}}\left(x, A_{1}\right) \in(0,1), \\
a_{1} \in P_{M_{1}}\left(x, A_{1}\right), \quad a_{2} \in P_{M_{2}}\left(x, A_{2}\right), \quad M_{1} \subset \mathfrak{B}_{R}, \\
\xi^{-}=h_{M_{1}}^{+}\left(M_{1}, M_{2}\right)<1, \quad \xi^{+}=h_{M_{1}}^{+}\left(M_{2}, M_{1}\right), \\
\eta^{-}=h_{M_{1}}\left(A_{1}, A_{2}\right), \quad \eta^{+}=h_{M_{1}}\left(A_{2}, A_{1}\right) .
\end{gathered}
$$

Тогда

$$
\left\|a_{1}-a_{2}\right\| \leqslant d_{1} \delta_{M_{1}}^{-1}(\tau)+\frac{R}{1-\xi^{-}}\left(d_{1}\left(\xi^{+}+\xi^{-}\right)+\eta^{-}\left(1+\xi^{+}\right)\right)
$$

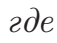

$$
\tau=\frac{d_{1}\left(\xi^{+}+\xi^{-}\right)+\eta^{-}\left(1+\xi^{+}\right)+\eta^{+}\left(1-\xi^{-}\right)}{2 \min \left\{d_{1},\left(1-d_{1}\right)\left(1-\xi^{-}\right)\right\}} .
$$

ДокАЗАтельство. Поскольку $a_{2} \in A_{2}$, то $d_{M_{1}}\left(a_{2}, A_{1}\right) \leqslant h_{M_{1}}^{+}\left(A_{2}, A_{1}\right)=\eta^{+}$. ОбозНачим

$$
d_{1}^{\prime}=\frac{1+\xi^{+}}{1-\xi^{-}}\left(d_{1}+\eta^{-}\right), \quad z=\frac{x-a_{1}}{d_{1}}, \quad w=\frac{x-a_{2}}{d_{1}^{\prime}} .
$$

Тогда

$\mu_{M_{1}}\left(x-a_{2}\right) \leqslant\left(1+\xi^{+}\right) \mu_{M_{2}}\left(x-a_{2}\right)=\left(1+\xi^{+}\right) d_{M_{2}}\left(x, A_{2}\right) \leqslant \frac{1+\xi^{+}}{1-\xi^{-}} d_{M_{1}}\left(x, A_{2}\right) \leqslant d_{1}^{\prime}$ и, следовательно, $w \in M_{1} \subset \mathfrak{B}_{R}$. Замечая, что

$$
0 \leqslant d_{1}^{\prime}-d_{1}=\frac{d_{1}\left(\xi^{+}+\xi^{-}\right)+\eta^{-}\left(1+\xi^{+}\right)}{1-\xi^{-}}, \quad \frac{d_{1}^{\prime}-d_{1}+\eta^{+}}{2 \min \left\{d_{1}^{\prime}, 1-d_{1}\right\}} \leqslant \tau
$$

и применяя лемму 7.9 для $A=A_{1}, M=M_{1}$, получаем доказываемое утверждение. 
Лемма 7.11. Пусть равномерно выпуклый квазишар $M \subset$ Е удовлетворяет включениям $\mathfrak{B}_{\sigma} \subset M \subset \mathfrak{B}_{\varkappa \sigma}$ при некоторых $\sigma, \varkappa \in(0,+\infty)$. Тогда для любых чисел $d>0$ и $t \in(0, d)$ справедливы неравенства

$$
\frac{d}{2} \delta_{M}^{-1}\left(\frac{t}{9 \varkappa d}\right)-\frac{3}{16} \sigma t \leqslant \theta_{M}(t, d, \text { Conv }) .
$$

ДокАЗАТЕЛьство. Зафиксируем произвольные числа $d>0$ и $t \in(0, d)$ и обозначим $\tau=\frac{t}{9 \varkappa d}$. Зафиксируем произвольное число $\varepsilon \in\left(0, \delta_{M}^{-1}(\tau)\right)$. В силу определения (5.10) модуля выпуклости найдутся векторы $x_{1}, x_{2} \in M$ такие, что

$$
\mu_{M}\left(x_{1}+x_{2}\right) \geqslant 2(1-\tau), \quad\left\|x_{1}-x_{2}\right\|>\varepsilon .
$$

Поэтому векторы

$$
x=\frac{x_{1}-x_{2}}{2}, \quad z=\frac{x_{1}+x_{2}}{2}, \quad y=\frac{\tau}{1-\tau} z
$$

удовлетворяют соотношениям

$$
z+x \in M, \quad z-x \in M, \quad z+y \notin \operatorname{int} M .
$$

Последнее соотношение следует из того, что

$$
\mu_{M}(z+y)=\frac{\mu_{M}(z)}{1-\tau}=\frac{\mu_{M}\left(x_{1}+x_{2}\right)}{2(1-\tau)} \geqslant 1 .
$$

Используя включения $z \in M \subset \mathfrak{B}_{\varkappa \sigma}$ и $\tau \in\left(0, \frac{1}{9 \varkappa}\right) \subset\left(0, \frac{1}{9}\right)$, получаем

$$
\|y\|=\frac{\tau}{1-\tau}\|z\| \leqslant \frac{9}{8} \tau\|z\| \leqslant \frac{9}{8} \tau \varkappa \sigma<\frac{\sigma}{8} .
$$

Рассмотрим отрезки

$$
A_{1}=[-z+x,-z-x-6 y], \quad A_{2}=[-z-x,-z+x-6 y] .
$$

В силу равномерной выпуклости квазишара $M$ множества $P_{M}\left(0, A_{i}\right)$ одноэлементны: $P_{M}\left(0, A_{i}\right)=\left\{a_{i}\right\}$, где $a_{1}=-z+x-t_{1}(x+3 y), a_{2}=-z-x+t_{2}(x-3 y)$ при некоторых $t_{i} \in[0,2], i=1,2$. Применяя лемму 7.3 для $A=A_{1}$ и используя неравенства $(7.42)$, имеем $t_{1} \leqslant \frac{1}{2}$ и

$$
\frac{3}{4} \leqslant 1-\frac{2\|y\|}{\sigma} \leqslant d_{M}\left(0, A_{1}\right) \leqslant 1 .
$$

Обозначим $d_{1}=d_{M}\left(0, A_{1}\right)$. Из неравенств (7.44) следует, что $d_{1} \in\left[\frac{3}{4}, 1\right]$.

Поскольку $a_{1}-a_{2}=\left(2-t_{1}-t_{2}\right) x+3 y\left(t_{2}-t_{1}\right)$, то $\left\|a_{1}-a_{2}\right\| \geqslant\|x\|-\frac{3}{2}\|y\|$. Используя соотношения (7.40)-(7.42), получаем неравенство

$$
\left\|a_{1}-a_{2}\right\| \geqslant \frac{1}{2} \varepsilon-\frac{27}{16} \tau \varkappa \sigma=\frac{1}{2} \varepsilon-\frac{3 \sigma \tau}{16 d} .
$$

Из равенств (7.43) и неравенства (7.42) следует, что

$$
h\left(A_{1}, A_{2}\right) \leqslant 6\|y\| \leqslant \frac{27 \tau \varkappa \sigma}{4}=\frac{3 \sigma \tau}{4 d} .
$$


Поэтому

$$
h_{M}\left(A_{1}, A_{2}\right) \leqslant \frac{1}{\sigma} h\left(A_{1}, A_{2}\right) \leqslant \frac{3 \tau}{4 d} .
$$

В силу определения (5.8) модуля $\theta_{M}$ имеем $\theta_{M}\left(\frac{3 \tau}{4 d}, d_{1}\right.$, Conv $) \geqslant\left\|a_{1}-a_{2}\right\|$. Поэтому согласно второму из равенств $(7.25)$, включению $d_{1} \in\left[\frac{3}{4}, 1\right]$ и неравенству (7.45) получаем

$$
\begin{aligned}
\theta_{M}(t, d, \text { Conv }) & =\frac{d}{d_{1}} \theta_{M}\left(\frac{t d_{1}}{d}, d_{1}, \text { Conv }\right) \geqslant d \theta_{M}\left(\frac{3 t}{4 d}, d_{1}, \text { Conv }\right) \\
& \geqslant d\left\|a_{1}-a_{2}\right\| \geqslant \frac{\varepsilon d}{2}-\frac{3}{16} \sigma \tau .
\end{aligned}
$$

Переходя к пределу при $\varepsilon \rightarrow \delta_{M}^{-1}(\tau)$, получаем доказываемое неравенство.

Лемма 7.12. Пусть равномерно выпукльй квазишар $M \subset E$ удовлетворяет включению $M \subset \mathfrak{B}_{R}$ при некотором $R>0$. Пусть $d>0, t \in(0,1)$. Тогда

$$
\frac{d}{2} \delta_{M}^{-1}(t)-\frac{R d t}{1-t} \leqslant \nu_{M}(t, d, \text { Conv })
$$

ДокАЗАтЕльство. Зафиксируем произвольное число $\varepsilon \in\left(0, \delta_{M}^{-1}(t)\right)$. Согласно определению (5.10) модуля выпуклости найдутся векторы $x_{1}, x_{2} \in M$ такие, что

$$
\mu_{M}\left(x_{1}+x_{2}\right) \geqslant 2(1-t), \quad\left\|x_{1}-x_{2}\right\|>\varepsilon .
$$

Обозначим

$$
y=\frac{x_{1}+x_{2}}{\mu_{M}\left(x_{1}+x_{2}\right)} .
$$

Поскольку $y \in \partial M$, по теореме Хана-Банаха об отделимости существует функционал $p \in E^{*}$ такой, что $\|p\|=1$ и

$$
\langle p, y\rangle=s(p, M):=\sup _{x \in M}\langle p, x\rangle .
$$

Заметим, что

$$
\frac{\left\langle p, x_{1}+x_{2}\right\rangle}{2}=\frac{\mu_{M}\left(x_{1}+x_{2}\right)}{2}\langle p, y\rangle \geqslant(1-t) s(p, M) .
$$

Предположим для определенности, что $\left\langle p, x_{1}\right\rangle \geqslant\left\langle p, x_{2}\right\rangle$. Тогда

$$
(1-t) s(p, M) \leqslant\left\langle p, x_{1}\right\rangle \leqslant s(p, M)=\langle p, y\rangle .
$$

Рассмотрим квазишар

$$
M^{\prime}=\left\{x \in M \mid\langle p, x\rangle \leqslant\left\langle p, x_{1}\right\rangle\right\}
$$

и замкнутое полупространство

$$
A=\{x \in E \mid\langle p, x\rangle \leqslant-d\langle p, y\rangle\} .
$$

Обозначим

$$
\xi=\frac{\langle p, y\rangle}{\left\langle p, x_{1}\right\rangle}, \quad a=-d y, \quad a^{\prime}=-\xi d x_{1}
$$


Если $x \in \operatorname{int}(d \cdot M), w \in A$, то $\langle p, x\rangle\langle d s(p, M)=d\langle p, y\rangle \leqslant-\langle p, w\rangle$. Поэтому $A \cap(-\operatorname{int}(d \cdot M))=\varnothing$ и, следовательно, $d_{M}(0, A) \geqslant d$. Используя соотношения $a=-d y \in A \cap(-d M)$, получаем равенство

$$
d_{M}(0, A)=d
$$

и включение

$$
a \in P_{M}(0, A) \text {. }
$$

Если $w \in A, x \in \operatorname{int}\left(\xi d M^{\prime}\right)$, то $\langle p, x\rangle\left\langle\xi d\left\langle p, x_{1}\right\rangle \leqslant d\langle p, y\rangle \leqslant-\langle p, w\rangle\right.$. Поэтому $A \cap\left(-\operatorname{int}\left(\xi d M^{\prime}\right)\right)=\varnothing$ и $\varrho_{M^{\prime}}(0, A) \geqslant \xi d$. Используя соотношения $a^{\prime}=-\xi d x_{1} \in$ $A \cap(-\xi d M)$, получаем включение

$$
a^{\prime} \in P_{M^{\prime}}(0, A) \text {. }
$$

В силу соотношений (7.49) и (7.51) справедливы неравенства $1 \leqslant \xi \leqslant \frac{1}{1-t}$. Используя соотношения (7.46), (7.47), получаем

$$
\left\|y-\frac{\xi\left(x_{1}+x_{2}\right)}{2}\right\|=\|y\|\left|1-\frac{\xi \mu_{M}\left(x_{1}+x_{2}\right)}{2}\right| \leqslant\|y\|\left|1-\frac{1}{1-t}\right| \leqslant \frac{R t}{1-t} .
$$

Следовательно, согласно равенствам (7.51) и второму неравенству формулы (7.46), приходим к соотношениям

$$
\left\|a-a^{\prime}\right\|=\left\|d y-\xi d x_{1}\right\| \geqslant \xi d\left\|\frac{x_{1}+x_{2}}{2}-x_{1}\right\|-d\left\|y-\frac{\xi\left(x_{1}+x_{2}\right)}{2}\right\| \geqslant \frac{\varepsilon d}{2}-\frac{R d t}{1-t} .
$$

Из соотношений (7.49), (7.50) следуют включения $(1-t) M \subset M^{\prime} \subset M$. Поэтому согласно равенству (5.7) имеем $h_{M}\left(M^{\prime}, M\right) \leqslant t$. Используя соотношения (7.52)-(7.55) и определение (5.9) модуля $\nu_{M}$, приходим к неравенству

$$
\nu_{M}(t, d, \text { Conv }) \geqslant\left\|a-a^{\prime}\right\| \geqslant \frac{\varepsilon d}{2}-\frac{R d t}{1-t} .
$$

Переходя к пределу при $\varepsilon \rightarrow \delta_{M}^{-1}(t)$, получаем доказываемое неравенство.

Лемма 7.13. Пусть $p \in(1,+\infty), E=L_{p}, \operatorname{dim} E \geqslant 3$. Тогда

$$
\Phi(t) \geqslant\left\{\begin{array}{lll}
\left(\frac{2}{p(p-1)}\right)^{1 / 2} t^{p / 2}+o\left(t^{p / 2}\right), & p \in(1,2), & \\
\left(\frac{p(p-1)}{2}\right)^{1 / p} t^{2 / p}+o\left(t^{2 / p}\right), & p \geqslant 2, &
\end{array} \rightarrow+0 .\right.
$$

ДокАЗАТЕЛЬСтво. Для любого числа $t \in[0,1]$ определим

$$
\lambda(t)=\left(\frac{1-t^{p}}{2}\right)^{1 / p}
$$

Покажем, что существуют число $t_{0} \in\left(0, \frac{1}{2}\right)$ и функция $\tau:\left[0, t_{0}\right] \rightarrow\left[0, \frac{1}{2}\right]$ такие, что

$$
\begin{gathered}
(\lambda(t)+\tau(t))^{p}+(\lambda(t)-\tau(t))^{p}=1 \quad \forall t \in\left[0, t_{0}\right], \\
\tau(t)=C t^{p / 2}+o\left(t^{p / 2}\right), \quad t \rightarrow+0,
\end{gathered}
$$

где $C=2^{-1 / p}\left(\frac{2}{p(p-1)}\right)^{1 / 2}$. 
Действительно, рассмотрим функцию

$$
\varphi(u, v)=\left(\left(\frac{1-u}{2}\right)^{1 / p}+v^{1 / 2}\right)^{p}+\left(\left(\frac{1-u}{2}\right)^{1 / p}-v^{1 / 2}\right)^{p}-1,
$$

где $u \in\left[0, \frac{1}{2}\right], v \in\left[0, \frac{1}{16}\right]$. Поскольку функция $\varphi:\left[0, \frac{1}{2}\right] \times\left[0, \frac{1}{16}\right] \rightarrow \mathbb{R}$ непрерывно дифференцируема,

$$
\varphi(0,0)=0, \quad \frac{\partial \varphi}{\partial u}(0,0)=-1, \quad \frac{\partial \varphi}{\partial v}(0,0)=4^{1 / p} \frac{p(p-1)}{2}=\frac{1}{C^{2}}>0,
$$

по теореме о неявной функции существуют число $u_{0} \in\left(0, \frac{1}{2}\right)$ и непрерывно дифференцируемая функция $v:\left[0, u_{0}\right] \rightarrow\left[0, \frac{1}{16}\right]$ такие, что

$$
\varphi(u, v(u))=0 \quad \forall u \in\left[0, u_{0}\right]
$$

$v(0)=0, v^{\prime}(0)=-\frac{\frac{\partial \varphi}{\partial u}(0,0)}{\frac{\partial \varphi}{\partial v}(0,0)}=C^{2}$. Следовательно,

$$
v(u)=C^{2} u+o(u), \quad u \rightarrow+0 .
$$

Обозначая $t_{0}=u_{0}^{1 / p}, \tau(t)=\left(v\left(t^{p}\right)\right)^{1 / 2}$ и используя равенства $(7.59),(7.60)$ при $u=t^{p}$, получаем равенства $(7.57),(7.58)$.

Поскольку $\operatorname{dim} E \geqslant 3$, существуют векторы $e_{1}, e_{2}, e_{3} \in E$, удовлетворяющие условию

$$
\left\|\sum_{i=1}^{3} \alpha_{i} e_{i}\right\|=\left(\sum_{i=1}^{3}\left|\alpha_{i}\right|^{p}\right)^{1 / p} \quad \forall \alpha_{1}, \alpha_{2}, \alpha_{3} \in \mathbb{R} .
$$

Для любого $t \in\left[0, t_{0}\right]$ рассмотрим векторы

$$
z_{t}=\lambda(t)\left(e_{1}+e_{2}\right), \quad x_{t}=\tau(t)\left(e_{1}-e_{2}\right), \quad y_{t}=t e_{3} .
$$

В силу равенств $(7.56),(7.57),(7.61)$ для любого числа $t \in\left[0, t_{0}\right]$ имеем

$$
\begin{gathered}
\left\|z_{t}+y_{t}\right\|=\left\|z_{t}-y_{t}\right\|=\left(2 \lambda^{p}(t)+t^{p}\right)^{1 / p}=1 \\
\left\|z_{t}+x_{t}\right\|=\left\|z_{t}-x_{t}\right\|=\left((\lambda(t)+\tau(t))^{p}+(\lambda(t)-\tau(t))^{p}\right)^{1 / p}=1 .
\end{gathered}
$$

Поэтому согласно определению (2.15) модуля $\Phi$ справедливы неравенства

$$
\Phi\left(\left\|y_{t}\right\|\right) \geqslant\left\|x_{t}\right\|, \quad \Phi\left(\left\|x_{t}\right\|\right) \geqslant\left\|y_{t}\right\| \quad \forall t \in\left[0, t_{0}\right] .
$$

Из равенств (7.58), (7.61), (7.62) вытекают соотношения

$$
\left\|y_{t}\right\|=t, \quad\left\|x_{t}\right\|=\tau(t) 2^{1 / p}=\left(\frac{2}{p(p-1)}\right)^{1 / 2} t^{p / 2}+o\left(t^{p / 2}\right), \quad t \rightarrow+0 .
$$

Таким образом, используя первое из неравенств формулы (7.63) в случае $p \in$ $(1,2)$ и второе из неравенств этой формулы в случае $p \geqslant 2$, получаем требуемое утверждение. 


\section{§ 8. Доказательство основных результатов}

ДоказАТЕЛЬСтво теоремы 6.1. Первое из неравенств (6.1) следует из леммы 7.5. Второе из этих неравенств вытекает из замечания 5.6. Третье неравенство справедливо согласно лемме 7.2.

ДокАЗАТЕЛЬСтво СЛЕДСТвия 6.1. Покажем, что

$$
\omega_{M}(t, d, \text { Conv }) \geqslant t \quad \forall t>0, \quad \forall d>0 .
$$

Зафиксируем произвольные числа $t>0$ и $d>0$. Рассмотрим произвольную прямую $A \subset E$ и точку $x_{1} \in E$ такие, что $d_{M}\left(x_{1}, A\right)=d$. Выберем точку $a_{1} \in$ $P_{M}\left(x_{1}, A\right)$ и точку $a_{2} \in A$ такие, что $\left\|a_{1}-a_{2}\right\|=t$. Тогда точка $x_{2}=x_{1}+a_{2}-a_{1}$ удовлетворяет условиям $a_{2} \in P_{M}\left(x_{2}, A\right)$ и $\left\|x_{1}-x_{2}\right\|=t$. Поэтому согласно определению (5.6) модуля $\omega_{M}$ получаем неравенство (8.1).

Из неравенства (8.1) и первого из неравенств (6.1) следует, что

$$
d \Phi_{M}\left(\frac{t}{8 d}\right) \leqslant \frac{17}{8} \omega_{M}(t, d, \text { Conv }) \quad \forall d \in(0,1), \quad \forall t \in(0, \sigma d) .
$$

Поскольку для любого числа $t \in\left(0, \frac{\operatorname{diam} M}{2}\right)$ найдутся векторы $z_{1}, z_{2} \in \partial M$ такие, что $\left\|z_{1}-z_{2}\right\|=2 t$, то, полагая $z=\frac{z_{1}+z_{2}}{2}, x=y=\frac{z_{1}-z_{2}}{2}$ согласно определению (5.13) модуля $\Phi_{M}$, получаем неравенство

$$
\Phi_{M}(t) \geqslant t \quad \forall t \in\left(0, \frac{\operatorname{diam} M}{2}\right) .
$$

Отсюда в силу последнего из неравенств (6.1) следует, что

$$
\omega_{M}(t, d, \mathcal{W C}(M)) \leqslant 4 d \Phi_{M}\left(\frac{(1+\varkappa) t}{2 \min \{d, 1-d\}}\right) \quad \forall d \in(0,1), \quad \forall t \in(0, \sigma d) .
$$

В силу второго из неравенств (6.1) и неравенств (8.2), (8.3) получаем доказываемое утверждение.

ДокАЗАТЕЛЬСтво ЛЕммы 6.1. Неравенства (6.2) получаются в результате применения лемм 7.6, 7.7. Соотношения (6.3) следуют из неравенств (6.2) и неравенства $\Omega_{M}(t) \geqslant t$, которое справедливо при всех $t \in\left(0, \frac{\operatorname{diam} M}{2}\right)$, что следует из определения (5.12) функции $\Omega_{M}$.

ДокАЗАТЕЛЬство лЕммы 6.2. Зафиксируем произвольные числа $t \in\left(0, \frac{\sigma}{2}\right]$ и $\varepsilon>0$. В силу определения (5.13) модуля $\Phi_{M}$ существуют векторы $x, y, z \in E$ такие, что

$$
\begin{gathered}
z+x \in \partial M, \quad z-x \in \partial M, \quad z+y \notin \operatorname{int} M, \quad z-y \notin \operatorname{int} M, \\
\|y\| \leqslant t, \quad\|x\|>\Phi_{M}(t)-\varepsilon .
\end{gathered}
$$

Используя замечание 5.3, получаем $\mu_{M}(z) \geqslant \mu_{M}(z+y)-\frac{\|y\|}{\sigma} \geqslant 1-\frac{t}{\sigma} \geqslant \frac{1}{2}$. Из включений $z+x \in \partial M, z-x \in \partial M$ следует, что $z \in M$, а значит, $\mu_{M}(z) \leqslant 1$. Итак,

$$
\mu_{M}(z) \in\left[\frac{1}{2}, 1\right] .
$$


Обозначим $z^{\prime}=\frac{z}{\mu_{M}(z)}, y^{\prime}=\frac{y}{\mu_{M}(z)}$. Поскольку $z+y \notin \operatorname{int} M, z-y \notin \operatorname{int} M$, то

$$
\begin{aligned}
\mu_{M}\left(z^{\prime}+y^{\prime}\right)+\mu_{M}\left(z^{\prime}-y^{\prime}\right)-2 & =\frac{\mu_{M}(z+y)-\mu_{M}(z-y)}{\mu_{M}(z)}-2 \\
& \geqslant \frac{2}{\mu_{M}(z)}-2 \geqslant 2\left(1-\mu_{M}(z)\right) .
\end{aligned}
$$

Используя включение $z^{\prime} \in \partial M$ и определение (5.11) модуля гладкости, имеем

$$
\varrho_{M}(2 t) \geqslant \varrho_{M}\left(\left\|y^{\prime}\right\|\right) \geqslant 1-\mu_{M}(z) .
$$

Поскольку $z+x \in \partial M$ и $z-x \in \partial M$, по определению (5.10) модуля выпуклости получаем $\delta_{M}^{-1}\left(1-\mu_{M}(z)\right) \geqslant 2\|x\|$. Таким образом,

$$
2\left(\Phi_{M}(t)-\varepsilon\right)<2\|x\| \leqslant \delta_{M}^{-1}\left(1-\mu_{M}(z)\right) \leqslant \delta_{M}^{-1}\left(\varrho_{M}(2 t)\right) .
$$

Переходя к пределу при $\varepsilon \rightarrow+0$, получаем доказываемое неравенство.

ДокАЗАТЕЛЬСтво теоремы 6.2. Первое из неравенств цепочки (6.5) следует из леммы 7.11, второе - из замечания 5.6, третье - из леммы 7.10, примененной для $M_{1}=M_{2}=M, d_{1}=d, \xi^{-}=\xi^{+}=0, \eta^{-}=\eta^{+}=t$.

ДокАЗАТЕЛЬСТво СЛЕДСТВИЯ 6.2. Поскольку квазишар $M$ ограничен, для некоторых положительных чисел $\sigma$ и $\varkappa$ он удовлетворяет включениям $\mathfrak{B}_{\sigma} \subset$ $M \subset \mathfrak{B}_{\varkappa \sigma}$.

Фиксируя произвольную точку $x \in \partial M$ и произвольное число $\varepsilon \in(0, \sigma]$ и полагая $y=\left(1-\frac{\varepsilon}{\|x\|}\right) x$, получаем $\|x-y\|=\varepsilon, \mu(x+y)=2-\frac{\varepsilon}{\|x\|} \geqslant 2-\frac{\varepsilon}{\sigma}$. Следовательно, согласно определению (5.10) модуля выпуклости имеем $\delta_{M}(\varepsilon) \leqslant$ $1-\frac{\mu_{M}(x+y)}{2} \leqslant \frac{\varepsilon}{2 \sigma}$. Поэтому

$$
\delta_{M}^{-1}(\tau) \geqslant 2 \sigma \tau \quad \forall \tau \in\left[0, \frac{1}{2}\right] .
$$

Зафиксируем произвольные числа $t>0$ и $d>0$. Выберем точки $a_{1}, a_{2}, x \in E$ такие, что $t=\mu_{M}\left(a_{2}-a_{1}\right) \geqslant \mu_{M}\left(a_{1}-a_{2}\right), \mu_{M}\left(x-a_{1}\right)=d$. В силу замечания 5.3 справедливо неравенство $\mu_{M}\left(a_{2}-a_{1}\right) \leqslant \frac{\left\|a_{1}-a_{2}\right\|}{\sigma}$. Для одноэлементых множеств $A_{1}=\left\{a_{1}\right\}, A_{2}=\left\{a_{2}\right\}$ имеем $d_{M}\left(x, A_{1}\right)=d, h_{M}\left(A_{1}, A_{2}\right)=t$. Поэтому согласно определению (5.8) модуля $\theta_{M}$ получаем

$$
\theta_{M}(t, d, \text { Conv }) \geqslant\left\|a_{1}-a_{2}\right\| \geqslant \sigma t \quad \forall t>0, \quad d>0 .
$$

Используя неравенства (8.5), (8.6) и цепочку неравенств (6.5), получаем доказываемые соотношения.

ДоКАЗАТЕЛЬСтво тЕОРЕмы 6.3. Из леммы 7.12 следует первое из неравенств (6.6). Второе из этих неравенств вытекает из замечания 5.6. Третье получается в результате применения леммы 7.10 для $M_{1}=M, A_{1}=A_{2}, d_{1}=d$, $\xi^{-}=\xi^{+}=t, \eta^{-}=\eta^{+}=0$.

ДоКАЗАТЕЛЬСТВо СЛЕДСТВИЯ 6.3. Поскольку квазишар $M$ ограничен, для некоторых положительных чисел $\sigma$ и $\varkappa$ он удовлетворяет включениям $\mathfrak{B}_{\sigma} \subset$ $M \subset \mathfrak{B}_{\varkappa \sigma}$. 
Зафиксируем произвольное число $t>0$ и точку $a \in \partial M$. Пусть $A$ - некоторая прямая, лежащая в касательной гиперплоскости к множеству $M$ в точке $a$. Зафиксируем точку $a^{\prime} \in A$ такую, что $\left\|a-a^{\prime}\right\|=\sigma t$. Пусть $M^{\prime}-$ выпуклая оболочка множества $M \cup\left\{a^{\prime}\right\}$. Согласно замечанию 5.3 справедливо неравенство $d_{M}\left(a^{\prime}, M\right) \leqslant \frac{\left\|a-a^{\prime}\right\|}{\sigma}=t$. Следовательно, $h_{M}\left(M^{\prime}, M\right) \leqslant t$. Поскольку $-a \in P_{M}(0,-A),-a^{\prime} \in P_{M^{\prime}}(0,-A), d_{M}(0,-A)=1$, в силу определения $(5.9)$ модуля $\nu_{M}$ справедливо неравенство $\nu_{M}(t, 1, \mathrm{Conv}) \geqslant \sigma t$. Учитывая второе из равенств (7.26), получаем

$$
\nu_{M}(t, d, \text { Conv }) \geqslant \sigma d t \quad \forall t>0, \quad d>0 .
$$

Используя неравенства (8.5), (8.7) и цепочку неравенств (6.6), получаем доказываемые соотношения.

Докажем теперь результаты из $\S 3,4$.

ДоКАЗАТЕЛЬСТВо ТЕОРЕмЫ 3.1. Применяя теорему 6.1 для $M=\mathfrak{B}_{1}, \sigma=$ $\varkappa=1$ и используя замечания $5.7,5.9$, для любых $d \in(0,1), t \in(0, d)$ имеем

$$
d \Phi\left(\frac{t}{8 d}\right)-\frac{9}{8} t \leqslant \omega(t, d, \text { Conv }) \leqslant \omega(t, d, \mathcal{P} \mathcal{R}(1)) \leqslant 2 d \Phi\left(\frac{t}{\min \{d, 1-d\}}\right)+2 t .
$$

Подставляя в эти неравенства $\frac{t}{r}$ вместо $t$ и $\frac{d}{r}$ вместо $d$ и умножая полученные неравенства на $r$, в силу равенств (7.24) получаем доказываемые оценки.

ДоКАЗАТЕЛЬСтво СЛЕДСТВИЯ 3.1. Применяя следствие 6.1 для $M=\mathfrak{B}_{1}$ и используя замечания $5.7,5.9$, для любого $d \in(0,1)$ имеем

$$
\omega(t, d, \mathcal{P} \mathcal{R}(1)) \asymp \omega(t, d, \text { Conv }) \asymp \Phi(t), \quad t \rightarrow+0 .
$$

Подставляя в эти соотношения $\frac{t}{r}$ вместо $t$ и $\frac{d}{r}$ вместо $d$, в силу равенств $(7.24)$ получаем доказываемые соотношения.

ДокАЗАТЕЛЬСТВА лЕмм 3.1, 3.2 получаются в результате применения лемм $6.1,6.2$ для $M=\mathfrak{B}_{1}, \sigma=\varkappa=1$ с учетом замечания 5.9.

ДокАЗАТЕЛЬСтво ЛЕммы 3.3. Используя результаты Ханнера для пространства $L_{p}$ (см. [26] и [27, раздел 1.е]), получаем $\delta(t) \asymp t^{2}, \varrho(t) \asymp t^{p}$ при $p \in(1,2]$ и $\delta(t) \asymp t^{p}, \varrho(t) \asymp t^{2}$ при $p \in[2,+\infty), t \rightarrow+0$. Следовательно, $\delta^{-1}(\varrho(t)) \asymp t^{\alpha}$ при $t \rightarrow+0$, где $\alpha=\min \left\{\frac{p}{2}, \frac{2}{p}\right\}$. Применяя леммы 3.2, 7.13, получаем доказываемое утверждение.

ДоКАЗАТЕЛЬСТво ТЕОРЕМЫ 3.2. Согласно условиям теоремы существуют числа $C_{1}, C_{2}>0$ такие, что $\delta(t) \geqslant C_{1} t^{q}$ при $t \in[0,2]$ и $\varrho(t) \leqslant C_{2} t^{s}$ при $t \geqslant 0$. Обозначим $\alpha=\frac{s}{q}$. Тогда в силу леммы 3.2 имеем

$$
2 \Phi(t) \leqslant C_{3}(2 t)^{\alpha} \quad \forall t \in\left(0, \frac{1}{2}\right]
$$

где $C_{3}=\left(\frac{C_{2}}{C_{1}}\right)^{1 / q}$. Заметим, что в силу неравенств $(2.12)$ справедливо включение $\alpha \in(0,1]$. 
Зафиксируем число $r^{\prime} \in(0, r)$ и точки $x_{1}, x_{2} \in O\left(A, r^{\prime}\right)$. Согласно предложению 3.2 множества $P\left(x_{i}, A\right)$ одноэлементны, т.е. при некоторых $a_{i} \in E$ справедливы равенства $P\left(x_{i}, A\right)=\left\{a_{i}\right\}, i=1,2$. Обозначим

$$
d_{1}=d\left(x_{1}, A\right), \quad t=\left\|x_{1}-x_{2}\right\| .
$$

Рассмотрим случай $t<d_{1}$. В силу теоремы 3.1 и неравенства (8.8) получаем

$$
\left\|a_{1}-a_{2}\right\| \leqslant 2 d_{1} \Phi\left(\frac{t}{\min \left\{d_{1}, r-d_{1}\right\}}\right)+2 t \leqslant C_{3} d_{1}\left(\frac{2 t}{\min \left\{d_{1}, r-d_{1}\right\}}\right)^{\alpha}+2 t .
$$

Поскольку $d_{1}<r^{\prime}<r$, при $t<d_{1}$ справедливы неравенства

$$
\left\|a_{1}-a_{2}\right\| \leqslant C_{3} \max \left\{d_{1}^{1-\alpha}, \frac{d_{1}}{\left(r-d_{1}\right)^{\alpha}}\right\}(2 t)^{\alpha}+2 t<\frac{r C_{3}}{\left(r-r^{\prime}\right)^{\alpha}}(2 t)^{\alpha}+2 t \leqslant C t^{\alpha},
$$

где

$$
C=\frac{2^{\alpha} C_{3} r}{\left(r-r^{\prime}\right)^{\alpha}}+4 r^{1-\alpha}
$$

В случае $d_{1} \leqslant t \leqslant r$ получаем

$$
\begin{aligned}
\left\|a_{1}-a_{2}\right\| & \leqslant\left\|a_{1}-x_{1}\right\|+\left\|x_{1}-x_{2}\right\|+\left\|x_{2}-a_{2}\right\|=d\left(x_{1}, A\right)+d\left(x_{2}, A\right)+\left\|x_{1}-x_{2}\right\| \\
& \leqslant 2 d\left(x_{1}, A\right)+2\left\|x_{1}-x_{2}\right\|=2 d_{1}+2 t \leqslant 4 t \leqslant 4 r^{1-\alpha} t^{\alpha} \leqslant C t^{\alpha} .
\end{aligned}
$$

Итак, при $t \leqslant r$ справедливо неравенство $\left\|a_{1}-a_{2}\right\| \leqslant C t^{\alpha}$. Следовательно, оператор $x \mapsto P(x, A)$ удовлетворяет условию Гёльдера на множестве $O\left(A, r^{\prime}\right)$ с показателем $\alpha$.

ДОКАЗАТЕЛЬСТВо ТЕОРЕмЫ 4.1. Применяя теорему 6.2 для $M=\mathfrak{B}_{1}, \sigma=$ $\varkappa=1$, с учетом замечаний $5.7,5.9$ для любых $d \in(0,1), t \in(0, d)$ получаем

$$
\theta(t, d, \mathcal{P} \mathcal{R}(1)) \leqslant d \delta_{M}^{-1}\left(\frac{t}{\min \{d, 1-d\}}\right)+t .
$$

Подставляя в последнее неравенство $\frac{t}{r}$ вместо $t$ и $\frac{d}{r}$ вместо $d$ и умножая полученное неравенство на $r$, в силу первого из равенств (7.25) получаем доказываемое неравенство.

ДОКАЗАТЕЛЬСТВО СЛЕДСТВИЯ 4.1 состоит в применении теоремы 4.1, первого из неравенств предложения 4.1 и неравенства $\theta(t, d, \mathrm{Conv}) \leqslant \theta(t, d, \mathcal{P} \mathcal{R}(r))$, которое следует из включения $\operatorname{Conv} \subset \mathcal{P} \mathcal{R}(r)$.

ДОКАЗАТЕЛЬСТВО СЛЕДСТВИЯ 4.2. Поскольку модуль выпуклости пространства $E$ порядка $q$, существует число $C_{1}>0$ такое, что $\delta(t) \geqslant C_{1} t^{q}$ при $t \in[0,2]$. Пусть $x \in E, r>0, r^{\prime} \in(0, r), A_{1} \in \mathcal{P} \mathcal{R}(r), A_{2} \subset E, d_{1}=d\left(x, A_{1}\right) \leqslant r^{\prime}$, $a_{i} \in P\left(x, A_{i}\right), i=1,2$. Обозначим $t=h\left(A_{1}, A_{2}\right)$. Если $t<d_{1}$, то в силу равенства $(2.5)$ и теоремы 4.1 имеем

$$
\left\|a_{1}-a_{2}\right\| \leqslant \theta\left(t, d_{1}, \mathcal{P} \mathcal{R}(r)\right) \leqslant d_{1} \delta^{-1}\left(\frac{t}{\min \left\{d_{1}, r-d_{1}\right\}}\right)+t \leqslant C t^{\frac{1}{q}},
$$


где $C=\left(C_{1}^{-\frac{1}{q}}+3\right) r\left(r-r^{\prime}\right)^{-\frac{1}{q}}$. Если $d_{1} \leqslant t \leqslant r$, то

$$
\begin{aligned}
\left\|a_{1}-a_{2}\right\| & \leqslant\left\|a_{1}-x\right\|+\left\|x-a_{2}\right\|=d\left(x, A_{1}\right)+d\left(x, A_{2}\right) \\
& \leqslant 2 d\left(x, A_{1}\right)+h\left(A_{1}, A_{2}\right)=2 d_{1}+t \leqslant 3 t \leqslant C t^{\frac{1}{q}} .
\end{aligned}
$$

Таким образом, при $t \leqslant r$ справедливо неравенство $\left\|a_{1}-a_{2}\right\| \leqslant C t^{\frac{1}{q}}$.

ДоКАЗАТЕЛЬСтво теоремы 4.2. Применяя теорему 6.3 для $M=\mathfrak{B}_{1}, R=1$, с учетом замечания 5.7 для любых $d^{\prime} \in(0,1), \tau \in(0,1)$ имеем

$$
\nu_{\mathfrak{B}_{1}}\left(\tau, d^{\prime}, \mathcal{P} \mathcal{R}(1)\right) \leqslant d^{\prime} \delta^{-1}\left(\frac{d^{\prime} \tau}{\min \left\{d^{\prime},(1-\tau)\left(1-d^{\prime}\right)\right\}}\right)+\frac{2 d^{\prime} \tau}{1-\tau} .
$$

Подставляя сюда $\tau=\frac{t}{1-t}, d^{\prime}=\frac{d}{r}$ и используя замечание 5.10 и первое из равенств (7.26), получаем

$$
\begin{aligned}
\nu(t, d, \mathcal{P} \mathcal{R}(r)) & \leqslant \nu_{\mathfrak{B}_{1}}(\tau, d, \mathcal{P} \mathcal{R}(r))=r \nu_{\mathfrak{B}_{1}}\left(\tau, d^{\prime}, \mathcal{P} \mathcal{R}(1)\right) \\
& \leqslant d \delta^{-1}\left(\frac{d t}{\min \{(1-t) d,(1-2 t)(r-d)\}}\right)+\frac{2 d t}{1-2 t} .
\end{aligned}
$$

ДоКАЗАТЕЛЬСТВо СЛЕДСТВИЯ 4.3 состоит в применении теоремы 4.2, первого из неравенств предложения 4.2 и неравенства $\nu(t, d, \mathrm{Conv}) \leqslant \nu(t, d, \mathcal{P} \mathcal{R}(r))$, которое следует из включения $\operatorname{Conv} \subset \mathcal{P} \mathcal{R}(r)$.

\section{Список литературы}

1. Ya. I. Alber, Jen-Chih Yao, "On the projection dynamical systems in Banach spaces", Taiwanese J. Math., 11:3 (2007), 819-847.

2. B. O. Björnestàl, Continuity of the metric projection operator, preprint, Dept. of Math, TRITA-MAT, 20, Royal Institute of Technology, Stockholm, 1974, 348 pp.

3. B. O. Björnestàl, "Local Lipschitz continuity of the metric projection operator", Approximation theory, Papers, VIth semester, Stefan Banach Internat. Math. Center, Warsaw, 1975, Banach Center Publ., 4, PWN, Warsaw, 1979, 43-53.

4. В.И. Бердышев, "О модуле непрерывности оператора наилучшего приближения", Матем. заметки, 15:5 (1974), 797-808; англ. пер.: V. I. Berdyshev, "On the modulus of continuity of an operator of best approximation", Math. Notes, 15:5 (1974), 478-484.

5. В. И. Бердышев, "Варьирование нормы в задаче о наилучшем приближении", $M a$ тем. заметки, 29:2 (1981), 181-196; англ. пер.: V. I. Berdyshev, "Variation of the norms in the problem of best approximation", Math. Notes, 29:2 (1981), 95-103.

6. В.И. Бердышев, Устойчивость минимизации функиионалов и равномерная непрерывность метрической проекции, Дисс... . докт. физ.-матем. наук, Матем. ин-т им. В. А. Стеклова АН СССР, М., 1988.

7. Ya. I. Alber, A. I. Notik, "On some estimates for projection operators in Banach space", Comm. Appl. Nonlinear Anal., 2:1 (1995), 47-55.

8. Ya. I. Alber, "A bound for the modulus of continuity for metric projections in a uniformly convex and uniformly smooth Banach space", J. Approx. Theory, 85:3 (1996), 237-249.

9. J.-P. Penot, "Continuity properties of projection operators", J. Inequal. Appl., 2005:5 (2005), 509-521. 
10. H. Federer, "Curvature measures", Trans. Amer. Math. Soc., 93:3 (1959), 418-491.

11. J.-P. Vial, "Strong and weak convexity of sets and functions", Math. Oper. Res., 8:2 (1983), 231-259.

12. F. H. Clarke, R. J. Stern, P. R. Wolenski, "Proximal smoothness and lower- $C^{2}$ property", J. Convex Anal., 2:1-2 (1995), 117-144.

13. F. Bernard, L. Thibault, N. Zlateva, "Characterizations of prox-regular sets in uniformly convex Banach spaces", J. Convex Anal., 13:3-4 (2006), 525-559.

14. F. Bernard, L. Thibault, N. Zlateva, "Prox-regular sets and epigraphs in uniformly convex Banach spaces: Various regularities and other properties", Trans. Amer. Math. Soc., 363:4 (2011), 2211-2247.

15. М. В. Балашов, Г. Е. Иванов, "Слабо выпуклые и проксимально гладкие множества в банаховых пространствах", Изв. РАН. Сер. матем., 73:3 (2009), 23-66; англ. пер.: M. V. Balashov, G. E. Ivanov, "Weakly convex and proximally smooth sets in Banach spaces", Izv. Math., 73:3 (2009), 455-499.

16. V.V. Goncharov, F.F. Pereira, "Neighbourhood retractions of nonconvex sets in a Hilbert space via sublinear functionals", J. Convex Anal., 18:1 (2011), 1-36.

17. V. V. Goncharov, F.F. Pereira, "Geometric conditions for regularity in a time-minimum problem with constant dynamics", J. Convex Anal., 19:3 (2012), 631-669.

18. G. Colombo, V. V. Goncharov, B. S. Mordukhovich, "Well-posedness of minimal time problems with constant dynamics in banach spaces", Set-Valued Var. Anal., 18:3-4 (2010), 349-372.

19. Г. Е. Иванов, М. С. Лопушански, "О корректности задач аппроксимации и оптимизации для слабо выпуклых множеств и функций”, Фундамент. и прикл. матем., 18:5 (2013), 89-118.

20. G. E. Ivanov, "Weak convexity of sets and functions in a Banach space", J. Convex Anal., 22:2 (2015), 365-398.

21. T. Abatzoglou, "Continuity of metric projections in uniformly convex and uniformly smooth Banach spaces", J. Approx. Theory, 39:4 (1983), 299-307.

22. G. Nordlander, "The modulus of convexity in normed linear spaces", Ark. Mat., 4:1 (1960), 15-17.

23. G. E. Ivanov, "On well posed best approximation problems for a nonsymmetric seminorm", J. Convex Anal., 20:2 (2013), 501-529.

24. Б. Т. Поляк, “Теоремы существования и сходимость минимизирующих последовательностей в задачах на экстремум при наличии ограничений", Докл. AH CCCP, 166:2 (1966), 287-290; англ. пер.: В. T. Poljak, "Existence theorems and convergence of minimizing sequences in extremum problems with restrictions", Soviet Math. Dokl., 7 (1966), 72-75.

25. M. V. Balashov, D. Repovš, "Uniform convexity and the splitting problem for selections", J. Math. Anal. Appl., 360:1 (2009), 307-316.

26. O. Hanner, "On the uniform convexity of $L^{p}$ and $l^{p ", ~ A r k . ~ M a t ., ~ 3: 3 ~(1956), ~ 239-244 . ~}$

27. J. Lindenstrauss, L. Tzafriri, Classical Banach spaces: Function spaces, Ergeb. Math. Grenzgeb., 97, Springer-Verlag, Berlin-New York, 1979, x+243 pp.

ГРИГОРИЙ ЕВГЕНЬЕВИч ИвАНОВ

Поступило в редакцию

(Grigorit E. IVANOV)

22.04 .2014

Московский физико-технический институт

07.11 .2014

(государственный университет),

г. Долгопрудный Московской обл.

E-mail: givanov@mail.mipt.ru 